\title{
SiO line emission from C-type shock waves: interstellar jets and outflows
}

\author{
A. Gusdorf ${ }^{1,2}$, S. Cabrit ${ }^{3}$, D. R. Flower ${ }^{1}$, and G. Pineau des Forêts ${ }^{2,3,4}$ \\ 1 Physics Department, The University, Durham DH1 3LE, UK \\ e-mail: david.flower@durham.ac.uk \\ 2 Institut d'Astrophysique Spatiale (IAS), Bâtiment 121, 91405 Orsay, France \\ ${ }^{3}$ LERMA (UMR 8112 du CNRS), Observatoire de Paris, 61 avenue de l'Observatoire, 75014 Paris, France \\ ${ }^{4}$ Université Paris-Sud 11 and CNRS (UMR 8617), France
}

Received 23 October 2007 / Accepted 12 February 2008

\section{ABSTRACT}

\begin{abstract}
We study the production of $\mathrm{SiO}$ in the gas phase of molecular outflows, through the sputtering of Si-bearing material in refractory grain cores, which are taken to be olivine. We calculate also the rotational line spectrum of the SiO. The sputtering is driven by neutral particle impact on charged grains, in steady-state C-type shock waves, at the speed of ambipolar diffusion. The emission of the $\mathrm{SiO}$ molecule is calculated by means of an LVG code. A grid of models, with shock speeds in the range $20<v_{\mathrm{s}}<50 \mathrm{~km} \mathrm{~s}^{-1}$ and preshock gas densities $10^{4}<n_{\mathrm{H}}<10^{6} \mathrm{~cm}^{-3}$, has been generated. We compare our results with those of an earlier study (Schilke et al. 1997). Improvements in the treatment of the coupling between the charged grains and the neutral fluid lead to narrower shock waves and lower fractions of $\mathrm{Si}(\lessgtr 10 \%)$ being released into the gas phase. Erosion of grain cores is significant ( $\gtrsim 1 \%)$ only for C-type shock speeds $v_{\mathrm{s}}>25 \mathrm{~km} \mathrm{~s}^{-1}$, given the adopted properties of olivine. More realistic assumptions concerning the initial fractional abundance of $\mathrm{O}_{2}$ lead to $\mathrm{SiO}$ formation being delayed, so that it occurs in the cool, dense postshock flow. Good agreement is obtained with recent observations of $\mathrm{SiO}$ line intensities in the L1157 and L1448 molecular outflows. The inferred temperature, opacity, and $\mathrm{SiO}$ column density in the emission region differ significantly from those estimated by means of LVG "slab" models. The fractional abundance of $\mathrm{SiO}$ is deduced and found to be in the range $4 \times 10^{-8} \lesssim n(\mathrm{SiO}) / n_{\mathrm{H}} \lesssim 3 \times 10^{-7}$. Observed line profiles are wider than predicted and imply multiple, unresolved shock regions within the beam.
\end{abstract}

Key words. astrochemistry - atomic processes - magnetohydrodynamics (MHD) - molecular processes - radiative transfer shock waves

\section{Introduction}

Unlike CO, which is observed extensively in the interstellar medium of our own and other galaxies, its homologue $\mathrm{SiO}$ is observed principally in outflows associated with regions of star formation. This striking difference in behaviour is a consequence of the lower elemental abundance and the more complete depletion of silicon from the gas phase during grain formation. Both carbon and silicon form refractory materials - graphite and silicates - of which the cores of interstellar grains are believed to be composed; but the much higher elemental abundance of carbon, $n_{\mathrm{C}} / n_{\mathrm{H}}=3.55 \times 10^{-4}$, compared with silicon, $n_{\mathrm{Si}} / n_{\mathrm{H}}=3.37 \times$ $10^{-5}$ (Anders \& Grevesse 1989), leads to some of the carbon remaining in the gas phase.

The $\mathrm{SiO}$ molecule was first detected in the Galactic centre (Sgr B2) by Wilson et al. (1971) and subsequently in Ori A by Dickinson (1972). More recent observations of $\mathrm{SiO}$ in jets (see, for example, Bachiller et al. 1991; Martín-Pintado et al. 1992; Codella et al. 1999; Nisini et al. 2007) imply that, in these objects, at least some of the silicon has been restored to the gas phase; this can be achieved through sputtering of the grain material, probably in shock waves, which are features of the outflows. It has been known since the study by Draine et al. (1983) that grain ice-mantles can be eroded in C-type shock waves, owing to impact of neutral particles on charged grains at the ion-neutral drift speed, which is the speed of ambipolar diffusion. More recent work (Flower \& Pineau des Forêts 1995; Flower et al. 1996; Field et al. 1997; May et al. 2000) has shown that this process might result also in the partial erosion of the refractory grain cores. The simulations undertaken by May et al. of the sputtering of various silicates (forsterite, fayalite and olivine) by neutral atoms showed that $C$-shock speeds in excess of $30 \mathrm{~km} \mathrm{~s}^{-1}$ are necessary to erode a significant fraction (more than a few per cent) of these materials. On the other hand, it has since been recognized (Le Bourlot et al. 2002; Ciolek et al. 2004) that the speeds at which C-type shock waves can propagate are limited, both by the collisional dissociation of molecular hydrogen, which leads to a thermal runaway and the formation of a J-type shock wave, and by the ion magnetosonic speed, whose value is constrained by the inertia of the charged grains. Consequently, the efficacy of the erosion of silicates in C-type shock waves is restricted by the maximum $\mathrm{C}$-shock speed, which depends on the preshock density and transverse magnetic field strength, and on the fraction of charged grains (Flower \& Pineau des Forêts 2003).

In a previous study of $\mathrm{SiO}$ production in the interstellar medium, Schilke et al. (1997, henceforth Sch97) considered in detail the erosion of silicon from grain cores and from their mantles by C-type shock waves and the resulting $\mathrm{SiO}$ emission spectrum; this study remains the only one of its kind that has been published to date. In the intervening decade, there has been progress in our understanding of the sputtering process 
(May et al. 2000), the gas-phase chemistry of silicon (Le Picard et al. 2001), and the grain dynamics (Flower \& Pineau des Forêts 2003), and so it seems timely to revisit this topic. Recent observations of $\mathrm{SiO}$ in jets (Nisini et al. 2007) extend to higher rotational levels than previously and provide a further motivation for such an study. Accordingly, we have reconsidered the chemistry of silicon in steady-state, planar MHD shock waves, with a view to providing a grid of models which may serve in the analysis of current and future observations of rotational transitions of $\mathrm{SiO}$ in outflows. These models yield additional results relating to rovibrational transitions of $\mathrm{H}_{2}$ and rotational lines of $\mathrm{CO}$, as well as forbidden lines of atoms and atomic ions, including [Fe II] (cf. Giannini et al. 2006).

Grain-grain collisions and the sputtering of silicon in oblique C-type shock waves, in which the preshock magnetic field direction is inclined to the plane of the shock front, have been considered by Caselli et al. (1997). However, such simulations have yet to incorporate the gas-phase chemistry and the radiative cooling processes, in order to enable quantitative analyses of the spectral line observations to be made.

\section{The model}

We summarize below those developments, in both the MHD code and the data used and produced by the code, which are relevant to the modelling of the intensities and the profiles of the rotational lines of $\mathrm{SiO}$. We take as our baseline the study by $\mathrm{Sch} 97$. The reader is referred to the more recent papers cited below for details of the modifications.

\subsection{The dynamics of charged grains}

The main revision of the code itself concerns the treatment of the charge and of the dynamical effects of the grains. The charge distribution of both the grains and the "very small grains" (VSG), represented by polycyclic aromatic hydrocarbons in our model, are calculated, assuming that collisions with gas-phase particles (electrons, ions and neutrals) determine the charge; see Flower \& Pineau des Forêts (2003). As was mentioned in the Introduction, allowance for the mass density of the (mainly negatively) charged grains can reduce significantly the magnetosonic speed in the charged fluid

$c_{\mathrm{m}}^{2}=\frac{5 k_{\mathrm{B}}\left(T_{+}+T_{-}\right)}{3\left(\mu_{+}+\mu_{-}\right)}+\frac{B^{2}}{4 \pi\left(\rho_{+}+\rho_{-}\right)}$

where $T_{ \pm}$are the temperatures, $\mu_{ \pm}$are the mean masses and $\rho_{ \pm}$ are the mass densities of the positively and negatively charged fluids; $B$ is the magnetic field strength in the preshock gas. The magnetosonic speed is the maximum speed at which a C-type shock can propagate in the medium. Furthermore, the momentum transfer between the charged grains and the neutral fluid affects the ion-neutral drift speed and has consequences for the degree of sputtering of the grains within a C-type shock wave.

\subsection{Radiative cooling by $\mathrm{H}_{2}$}

The thermal balance of the medium, particularly the radiative cooling by $\mathrm{H}_{2}$, is treated more exactly in the current model. Rovibrational excitation of $\mathrm{H}_{2}$, principally by $\mathrm{H}, \mathrm{H}_{2}$ itself, and $\mathrm{He}$, followed by radiative decay, is the principal mechanism of cooling the shock-heated gas. Following Le Bourlot et al. (2002), the populations of the rovibrational levels of $\mathrm{H}_{2}$ are calculated in parallel with the hydrodynamical and chemical rate equations, yielding the most accurate determination of the rate of cooling by $\mathrm{H}_{2}$ that is achievable within the framework of a timeindependent (steady-state) model of the shock structure. The rate coefficients for the collisional excitation by $\mathrm{H}$ of rovibrational transitions of $\mathrm{H}_{2}$ are from the recent work of Wrathmall et al. (2007). Collisional dissociation and ionization of $\mathrm{H}_{2}$, as well as ionization of $\mathrm{H}$, are taken into account. Collisional dissociation of $\mathrm{H}_{2}$ is a particularly important process, as the removal of $\mathrm{H}_{2}$ can lead to a thermal runaway. The associated increase in the kinetic temperature, $T_{\mathrm{n}}$, of the neutral fluid, and hence of the adiabatic sound speed

$c_{\mathrm{s}}^{2}=\frac{5 k_{\mathrm{B}} T_{\mathrm{n}}}{3 \mu_{\mathrm{n}}}$

where $\mu_{\mathrm{n}}$ is the mean mass of the neutral fluid, can give rise to a sonic point in the flow and hence to a J-type discontinuity. This phenomenon imposes an additional constraint on the maximum speed of a C-type shock wave in a molecular medium.

In the compressed gas of the postshock region where most of $\mathrm{SiO}$ forms, cooling by ${ }^{12} \mathrm{CO},{ }^{13} \mathrm{CO}$, and $\mathrm{H}_{2} \mathrm{O}$ starts to dominate that by $\mathrm{H}_{2}$. In order to predict accurately the emitted $\mathrm{SiO}$ emission spectrum, an LVG treatment of the cooling by these species has been introduced, following the procedures of Neufeld \& Kaufman (1993). A "thermal gradient" $c_{\mathrm{S}} / z^{\prime}$, where $z^{\prime}=z+1.0 \times$ $10^{13} \mathrm{~cm}$ and $z$ is the independent integration variable, is added quadratically to the macroscopic velocity gradient, in order to simulate photon escape through thermal line broadening.

\subsection{The sputtering of grains}

The sputtering probabilities computed by May et al. (2000) for olivine $\left(\mathrm{MgFeSiO}_{4}\right)$ are used to determine the rate of erosion of $\mathrm{Si}$ from (charged) silicate grains by neutral particles. We include also the sputtering of carbonaceous (amorphous carbon) grains, using the sputtering yields of Field et al. (1997). Impacts (at the ion-neutral drift speed) of abundant heavy neutral species are the most effective in eroding the grain cores. For collisions with $\mathrm{CO}$, for example, we adopted the sputtering probabilities calculated for impacts of $\mathrm{Si}$ atoms, which have the same mass as $\mathrm{CO}$ and hence the same impact energy. As May et al. (2000) have shown, similar yields of $\mathrm{Si}$ are obtained from the three types of silicate: fayalite, $\mathrm{Fe}_{2} \mathrm{SiO}_{4}$; forsterite, $\mathrm{Mg}_{2} \mathrm{SiO}_{4}$; and olivine, $\mathrm{MgFeSiO}_{4}$.

The grain mantles are eroded first, through impacts with the most abundant neutral species, $\mathrm{H}, \mathrm{H}_{2}$ and $\mathrm{He}$, at ion-neutral drift speeds which are below the threshold for erosion of the cores (Draine et al. 1983; Flower \& Pineau des Forêts 1994). The initial composition of the gas is calculated in chemical equilibrium, whilst that of the grain mantles, and the elemental depletion into the grain cores, is based on observations (cf. Flower \& Pineau des Forêts 2003, Table 1). We incorporated a representative polycyclic aromatic hydrocarbon $(\mathrm{PAH})$, with a fractional abundance $n_{\mathrm{PAH}} / n_{\mathrm{H}}=10^{-6}$, an upper limit which is consistent with estimates of the fraction of elemental carbon likely to be present in the form of very small grains (Li \& Draine 2001; Weingartner \& Draine 2001). The state of charge of this species was computed following Flower \& Pineau des Forêts (2003), who showed that increasing the fractional abundance of the PAH results in higher values of the magnetosonic speed in the preshock gas, thereby enabling C-type shock waves to propagate at higher speeds. In their turn, the higher speed shocks erode the silicate grains more effectively. However, the adopted PAH abundance has little effect on the internal structure of the shock wave, as the grains become negatively charged in the shock wave and dominate grain-neutral momentum transfer. 
The total number density of the grains was computed

- from the total mass of the refractory cores, which is $0.78 \times$ $10^{-2}$ times the mass of the gas for the adopted composition of the cores;

- assuming a bulk mass density of $3 \mathrm{~g} \mathrm{~cm}^{-3}$; and

- taking a size distribution of the grain cores

$\mathrm{d} n_{\mathrm{g}}\left(a_{\mathrm{g}}\right) / \mathrm{d} a_{\mathrm{g}} \propto a_{\mathrm{g}}^{-3.5}$,

following Mathis et al. (1977), where $n_{\mathrm{g}}\left(a_{\mathrm{g}}\right)$ is the number density of grains with radius $a_{\mathrm{g}}$, assumed to have upper and lower limits of $0.3 \mu \mathrm{m}$ and $0.01 \mu \mathrm{m}$, respectively.

The thickness of the grain mantles was determined from their molecular composition (Flower \& Pineau des Forêts 2003, Table 2), assuming a mean number of $5 \times 10^{4}$ molecular binding sites per layer of the mantle and a thickness of $2 \times 10^{-4} \mu \mathrm{m}$ for each layer, independent of the size of the grain core; there are no $\mathrm{Si}$-containing species in the mantles. This procedure yields an initial mantle thickness of $0.015 \mu \mathrm{m}$ on the grain cores, whose mean radius is $a_{\mathrm{g}}=0.02 \mu \mathrm{m}$. However, the erosion of the grain mantles occurs sufficiently rapidly, as the ion and neutral flow velocities begin to decouple in the shock wave, that the existence of thick ice-mantles on the grains in the preshock gas has little effect on the shock dynamics (see Fig. 6 of Guillet et al. 2007).

\subsection{Chemistry}

The chemical reaction network comprises over 900 reactions connecting the abundances of more than 100 species, in both the gas and the solid phases. The complete list of species and reactions is available from http://massey.dur.ac.uk/drf/ outflows_test/species_chemistry_shock/. In the context of the present study, we emphasize the gas-phase chemistry of silicon and the formation of $\mathrm{SiO}$, in particular. The total rate of cosmic ray ionization of hydrogen, $\zeta$, was taken to be $5 \times$ $10^{-17} \mathrm{~s}^{-1}$.

Two reactions are important for the formation of $\mathrm{SiO}$ from $\mathrm{Si}$, which is released into the gas phase by the sputtering of the grains, namely

$$
\begin{aligned}
& \mathrm{Si}+\mathrm{O}_{2} \longrightarrow \mathrm{SiO}+\mathrm{O} \\
& \mathrm{Si}+\mathrm{OH} \longrightarrow \mathrm{SiO}+\mathrm{H}
\end{aligned}
$$

for which the following rate coefficients $\left(\mathrm{cm}^{3} \mathrm{~s}^{-1}\right)$

$$
\begin{aligned}
& k_{1}=2.7 \times 10^{-10} \exp (-111 / T) \\
& k_{2}=1.0 \times 10^{-10} \exp (-111 / T)
\end{aligned}
$$

were adopted by Sch97 from the compilation of Langer \& Glassgold (1990). The exponential factor in Eq. (3) derives from the argument (Graff 1989) that the reactions proceed only with the fine-structure states of $\mathrm{Si}\left(3 \mathrm{p}^{2}{ }^{3} \mathrm{P}_{J}\right)$ with $J>0$, of which $J=1$, which lies $111 \mathrm{~K}$ above the $J=0$ ground state, is the more significantly populated at low temperatures. More recently, the rate coefficient for reaction (1) has been measured at low temperatures $(15 \leq T \leq 300 \mathrm{~K})$ by Le Picard et al. (2001) and found to be given by

$k_{1}=1.72 \times 10^{-10}(T / 300)^{-0.53} \exp (-17 / T)$.

We have adopted (5) and the same expression for $k_{2}$. Evidently, the differences between the present and previous values of these rate coefficients are most significant for temperatures $T \lesssim 100 \mathrm{~K}$, i.e. in the cooling flow of the shocked gas.

The abundance of $\mathrm{SiO}$ is limited by its conversion to $\mathrm{SiO}_{2}$ in the reaction with $\mathrm{OH}$

$$
\mathrm{SiO}+\mathrm{OH} \longrightarrow \mathrm{SiO}_{2}+\mathrm{H}
$$

whose rate coefficient remains subject to considerable uncertainty. We adopt the same expression as Sch97, viz.

$k_{6}=1.0 \times 10^{-11}(T / 300)^{-0.7}$

in units of $\mathrm{cm}^{3} \mathrm{~s}^{-1}$. However, we note that Zachariah \& Tsang (1995) calculated a barrier of $433 \mathrm{~K}$ to reaction (6), and a rate coefficient

$k_{6}=2.5 \times 10^{-12}(T / 300)^{0.78} \exp (-613 / T) ;$

see the discussion of Le Picard et al. (2001). At $T=300 \mathrm{~K}$, the latter rate coefficient is 30 times smaller than the former. In the ambient (preshock) and the postshock gas, where $T \approx$ $10 \mathrm{~K}$, the existence of an activation energy of several hundred kelvin would prevent the oxidation of $\mathrm{SiO}$ in reaction (6) from occurring. The rate coefficient for reaction (6) in the UMIST data base (Le Teuff et al. 2000) is

$k_{6}=2.0 \times 10^{-12}$

in $\mathrm{cm}^{3} \mathrm{~s}^{-1}$, which is 50 times smaller than (7) at $T=10 \mathrm{~K}$. Fortunately, the conversion of $\mathrm{SiO}$ into $\mathrm{SiO}_{2}$ occurs in a region which is too cold and optically thick to contribute to the $\mathrm{SiO}$ line intensities, and so the uncertainty in the rate coefficient for reaction (6) is not significant in the present context.

Re-adsorption of molecules on to grains ("freeze-out") in the postshock gas is included, as in Sch97. The effects of freeze-out on the abundance of $\mathrm{SiO}$ and its spectrum are considered below.

\subsection{Line transfer}

The physical and chemical profiles which derive from the shock model summarized above are used in a large velocity gradient (LVG) calculation of the intensities of the emission lines of $\mathrm{SiO}$ and of CO. Our implementation of this technique is described in Appendix A, where we note that an expression for the escape probability which differs from that of Sch97 has been adopted.

\section{Results}

\subsection{Comparison with the calculations of Schilke et al. (1997)}

First, we compare the computed shock structure with that of Sch97, for a reference C-type shock model, in which the preshock density $n_{\mathrm{H}}=10^{5} \mathrm{~cm}^{-3}$ and the magnetic field strength $B=200 \mu \mathrm{G}$, and the shock velocity $v_{\mathrm{s}}=30 \mathrm{~km} \mathrm{~s}^{-1}$. The most striking difference between the current and the previous model is that the width of the shock wave decreases by a factor of approximately 4 , to $5 \times 10^{15} \mathrm{~cm}$, from $2 \times 10^{16} \mathrm{~cm}$ in the study of Sch97; see Fig. 1a. This difference is attributable to the more accurate treatment of the coupling between the neutral fluid and the charged grains in the current model and is an indication of the significance of the inertia of the (negatively) charged grains in dark clouds, in which the degree of ionization is low. With the narrower shock wave is associated a higher maximum temperature of the neutral fluid, as there is less time for the initial energy flux, $\rho_{\mathrm{n}} v_{\mathrm{s}}^{3} / 2$, associated with the bulk flow, to be converted into internal energy of the $\mathrm{H}_{2}$ molecules or to be radiated 

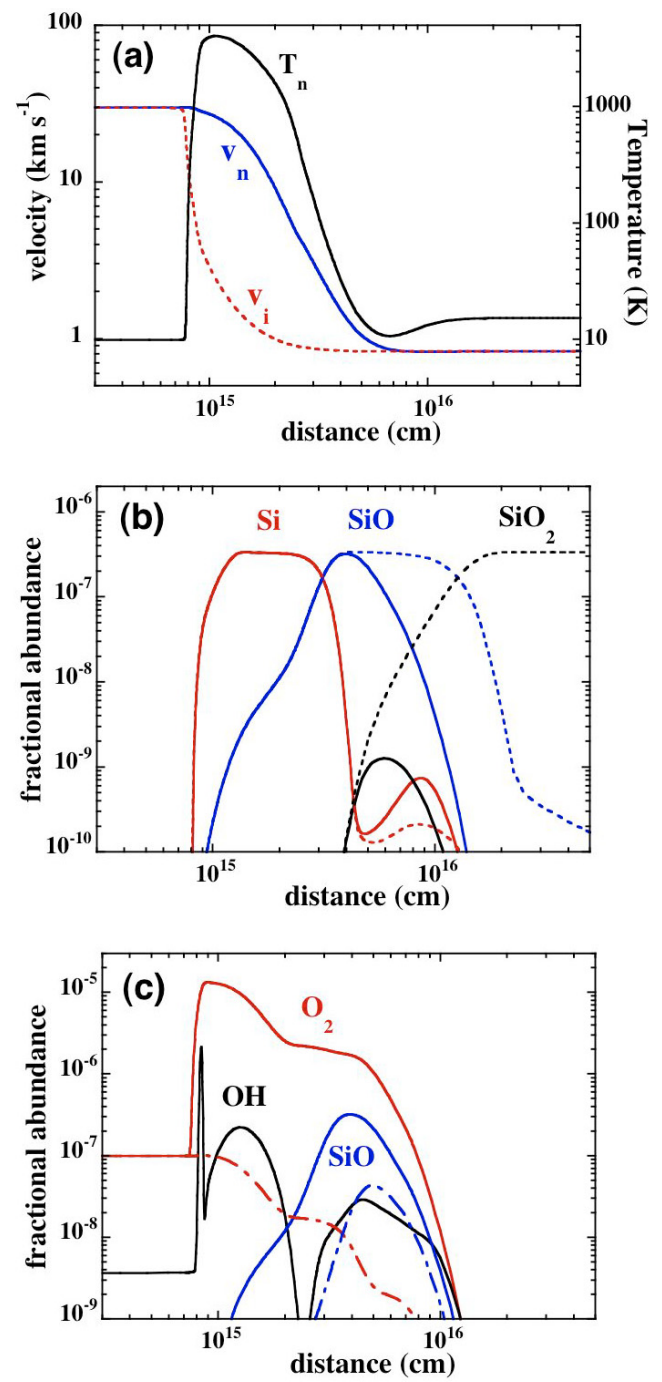

Fig. 1. a) Temperature of the neutral fluid and velocity profiles of the neutral and charged fluids, predicted by the present $\mathrm{C}$-type shock model. The shock parameters are $n_{\mathrm{H}}=10^{5} \mathrm{~cm}^{-3}$ and $B=200 \mu \mathrm{G}$ in the preshock gas, and $v_{\mathrm{s}}=30 \mathrm{~km} \mathrm{~s}^{-1}, \zeta=5 \times 10^{-17} \mathrm{~s}^{-1}$. The fractional gas-phase abundances of selected Si-and O-bearing species are plotted in panels b) and c); cf. Sch97, Fig. 2. The broken curves in panel b) are the corresponding results obtained when re-adsorption on to grains is neglected. The discontinuous curves in panel c) show the effects of assuming the initial abundance of $\mathrm{O}_{2}$ ice to be negligible, i.e. the second of the two scenarios described in Sect. 3.1.

away. Thus, $T_{\mathrm{n}} \approx 4000 \mathrm{~K}$ here, compared with $T_{\mathrm{n}} \approx 2000 \mathrm{~K}$ in the model of Sch97.

There are chemical differences between the models also, which are related in part to the changes in the shock structure; these differences may be summarized as follows.

(i) The fraction of the $\mathrm{Si}$ in the grain cores which is released into the gas phase by sputtering is approximately ten times smaller in the current model than in the model of Sch97. This reduction is attributable partly to the sputtering yields, which have higher thresholds and are smaller for olivine $\left(\mathrm{MgFeSiO}_{4}\right)$ than for the amorphous silica $\left(\mathrm{SiO}_{2}\right)$ considered by $\mathrm{Sch} 97$; but the main reason for the decrease is the enhanced coupling between the neutral fluid and the charged grains, which reduces the shock width and hence the time available to erode the grains. On the other hand, the magnitude of the ion-neutral drift speed is similar in both calculations. As a consequence of the reduction in the shock width, the integrated $\mathrm{SiO}$ line intensities predicted by the current model are smaller, in general, than calculated by Sch97; see Sect. 4.

(ii) The displacement of the maximum fractional abundance of $\mathrm{SiO}$, which forms in the gas-phase reactions (1) and (2), from that of $\mathrm{Si}$, which is eroded from the grains, is a more significant fraction of the shock width in the current model; cf. Fig. 1b. The initial fractional abundance of $\mathrm{O}_{2}$ in the preshock medium is lower here, by a factor of approximately 10, than in the model of Sch97, delaying the initial formation of $\mathrm{SiO}$. The $\mathrm{O}_{2}$ is assumed to be predominantly in the form of ice, which is sputtered rapidly from the grains in the early stages of development of the shock wave, as may be seen from the two orders of magnitude increase in the fractional abundance of gas-phase $\mathrm{O}_{2}$, apparent in Fig. 1c. The fractional abundances of $\mathrm{O}_{2}$ and $\mathrm{OH}$ decrease subsequently, at high kinetic temperatures, owing to their dissociation by $\mathrm{H}$ in the chemical reactions $\mathrm{O}_{2}(\mathrm{H}, \mathrm{O}) \mathrm{OH}$ and $\mathrm{OH}(\mathrm{H}, \mathrm{O}) \mathrm{H}_{2}$. The former reaction, which is endothermic by over $8000 \mathrm{~K}$, proves to be less effective in destroying $\mathrm{O}_{2}$ over the smaller width of the current shock model (see Fig. 1c) than was the case in the calculations of Sch97. On the other hand, the lower energy threshold of $17 \mathrm{~K}$ in reaction (5) allows oxidation reactions to proceed further into the postshock region, compared with Sch97, whose adopted threshold was $111 \mathrm{~K}$. As a consequence, conversion of $\mathrm{Si}$ into $\mathrm{SiO}$ is slower initially but more complete eventually than predicted by Sch97.

(iii) $\mathrm{SiO}_{2}$ is removed more rapidly from the gas phase in the current model. The compression is more rapid than in the model of Sch97, and so the rate of adsorption of molecules to grains ("freeze-out") is higher. If the oxidation of $\mathrm{SiO}$ in the reaction $\mathrm{SiO}(\mathrm{OH}, \mathrm{H}) \mathrm{SiO}_{2}$ has an activation energy of several hundred kelvin (Zachariah \& Tsang 1995; see Sect. 2.4), the maximum fractional abundance of $\mathrm{SiO}_{2}$ would be reduced still further.

Also shown in Fig. 1b are the fractional abundances which are obtained neglecting re-adsorption on to the grains. The timescale for freeze-out is sufficiently large that the chemical profiles are modified only in the cold postshock gas, where the flow speed is practically constant and the optical depths in the $\mathrm{SiO}$ lines are large. Consequently, whilst the effects re-adsorption on the composition of the postshock gas are dramatic, the freeze-out of $\mathrm{SiO}$ has no effect on the predicted line intensities.

In chemical equilibrium, the initial fractional abundance of $\mathrm{O}_{2}$ is approximately $10^{-5}$, whereas observations with the Odin satellite (Pagani et al. 2003; Larsson et al. 2007) have placed upper limits of $n\left(\mathrm{O}_{2}\right) / n\left(\mathrm{H}_{2}\right) \lesssim 10^{-7}$. In view of these measurements, we have considered two scenarios, both with an initial gas-phase fractional abundance $n\left(\mathrm{O}_{2}\right) / n_{\mathrm{H}}=1.0 \times 10^{-7}$, as a consequence of the freeze-out of oxygen on to grains, but with differing assumptions regarding its chemical form in the grain mantles.

- The molecular oxygen which formed in the gas phase was adsorbed on to the grains, where it remained as $\mathrm{O}_{2}$ ice in the preshock medium, with a fractional abundance of $1.3 \times 10^{-5}$, relative to $n_{\mathrm{H}}$. The initial fractional abundance of $\mathrm{H}_{2} \mathrm{O}$ ice is an order of magnitude larger than that the fractional abundance of $\mathrm{O}_{2}$ ice.

- Atomic oxygen was adsorbed on to the grains before $\mathrm{O}_{2}$ was synthesized and subsequently hydrogenated to $\mathrm{H}_{2} \mathrm{O}$ ice in the grain mantles of the cold preshock medium. The 
fractional abundance of $\mathrm{H}_{2} \mathrm{O}$ ice increases by only $25 \%$ as a consequence, to $1.3 \times 10^{-4}$.

It turns out that the first scenario is practically equivalent to assuming that the $\mathrm{O}_{2}$ is initially in the gas-phase (see Fig. 1c), as its release from the grain mantles occurs early and rapidly (on a timescale of a few years for the model in Fig. 1) in the shock wave. On the other hand, the second scenario can result in reduced levels of oxidation of $\mathrm{Si}$ to $\mathrm{SiO}$ in the gas-phase (cf. Fig. 1), depending on the relative importance of reactions 1 and 2 in the oxidation process. In what follows, we present results corresponding principally to the first scenario, with the second being considered mainly in Appendix C.

\subsection{A grid of models}

We have computed a grid of shock models with the following parameters:

$$
\begin{aligned}
& -n_{\mathrm{H}}=10^{4} \mathrm{~cm}^{-3}, v_{\mathrm{s}}=20,25,30,35,40,45,50 \mathrm{~km} \mathrm{~s}^{-1} \\
& -n_{\mathrm{H}}=10^{5} \mathrm{~cm}^{-3}, v_{\mathrm{s}}=20,25,30,35,40,45 \mathrm{~km} \mathrm{~s}^{-1} \\
& -n_{\mathrm{H}}=10^{6} \mathrm{~cm}^{-3}, v_{\mathrm{s}}=20,25,27,30,32,34 \mathrm{~km} \mathrm{~s}^{-1}
\end{aligned}
$$

In all of these models, we characterized the preshock magnetic field strength by

$B=b n_{\mathrm{H}}^{0.5}$,

where $n_{\mathrm{H}}$ is in $\mathrm{cm}^{-3}$ and $B$ is in $\mu \mathrm{G}$; the scaling parameter $b$ was taken equal to 1 . (The effect on $\mathrm{Si}$ sputtering of varying $b$ is discussed in Sect. 3.3.) The maximum shock speed for $n_{\mathrm{H}} \gtrsim 10^{4} \mathrm{~cm}^{-3}$ is determined by the collisional dissociation of $\mathrm{H}_{2}$, the main coolant, which leads to a thermal runaway and a J-discontinuity (Le Bourlot et al. 2002; Flower \& Pineau des Forêts 2003).

In fact, we computed two grids, one for each of the scenarios concerning the initial distribution of oxygen between $\mathrm{O}_{2}$ and $\mathrm{H}_{2} \mathrm{O}$ ices, as specified towards the end of the previous Sect. 3.1. We concentrate on the first of these two scenarios, but some additional figures for the second scenario are given in Appendix C as online material. (Our results are available in digital tabular format on request to the authors.)

Because of the sharply defined sputtering threshold energy of approximately $50 \mathrm{eV}$, there is negligible sputtering of $\mathrm{Si}$ from the olivine $\left(\mathrm{MgFeSiO}_{4}\right)$ for shock speeds of $20 \mathrm{~km} \mathrm{~s}^{-1}$ or less. The fractions of the $\mathrm{Mg}, \mathrm{Si}$ and $\mathrm{Fe}$ which are released from the olivine into the gas phase are shown in Fig. 2. Comparing Fig. 2 with the corresponding Fig. 4 of May et al. (2000), whose sputtering yields are used in the present calculations, shows that the fractions of $\mathrm{Mg}, \mathrm{Si}$ and $\mathrm{Fe}$ which are sputtered from olivine have decreased by an order of magnitude. As the same sputtering yields have been used in both studies, this change is attributable to the reduction in the shock width, resulting from the improved treatment of grain-neutral coupling. We note that $\mathrm{CO}$ is the principal eroding partner (cf. May et al. 2000).

Figure 2 shows that the degree of sputtering is, in fact, insensitive to the preshock gas density (cf. Caselli et al. 1997); it depends essentially on the shock speed. Polynomial fits of the sputtered fractions of $\mathrm{Fe}, \mathrm{Si}$ and $\mathrm{Mg}$, as functions of the shock speed, are given in Appendix B.

In Fig. 3, we display the fractional gas-phase abundances of $\mathrm{Si}$ and $\mathrm{SiO}$, as functions of the relevant flow time. Silicon is produced by erosion of the charged grains by collisions, principally with molecules, at the ion-neutral drift speed. Once the drift speed exceeds the sputtering threshold velocity, the erosion

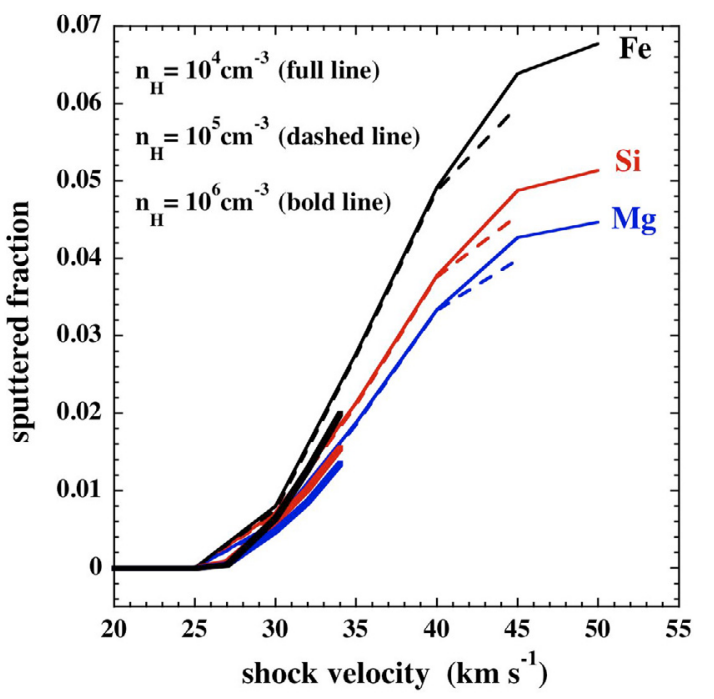

Fig. 2. The fractions of $\mathrm{Mg}, \mathrm{Si}$ and $\mathrm{Fe}$, initially in the form of olivine $\left(\mathrm{MgFeSiO}_{4}\right)$, which are released into the gas phase by sputtering within a steady-state $\mathrm{C}$-type shock wave.

of Si occurs rapidly, as Fig. 3 shows. Thus, the flow time which is directly relevant to the release of Si into the gas phase is that of the charged fluid, rather than that of the neutrals, which is the appropriate measure of the total time for formation of $\mathrm{SiO}$. As noted in item (ii) of Sect. 3.1, there is an additional, chemical delay to the conversion of $\mathrm{Si}$ into $\mathrm{SiO}$, in reactions (1) and (2), which is apparent in our Fig. 3, owing to the low abundance and partial destruction of $\mathrm{O}_{2}$. The magnitude of this delay depends on the parameters of the model, notably the shock speed, $v_{\mathrm{s}}$, and the preshock gas density, $n_{\mathrm{H}}$. Conversion is almost instantaneous for $v_{\mathrm{s}} \geq 30 \mathrm{~km} \mathrm{~s}^{-1}, n_{\mathrm{H}}=10^{6} \mathrm{~cm}^{-3}$, when $\mathrm{OH}$ is formed abundantly at the start of the shock and reaction (2) dominates the oxidation process.

Figure 4 shows the variation with shock speed and preshock gas density of the fractional abundance of $\mathrm{SiO}$, computed through the entire shock wave. It is evident from Fig. 4 that the duration of the C-type shock wave, as measured by the temperature profile, is of the order of $10^{4}, 10^{3}$ and $10^{2} \mathrm{yr}$ for preshock gas densities $n_{\mathrm{H}}=10^{4}, 10^{5}$ and $10^{6} \mathrm{~cm}^{-3}$, respectively. The peak $\mathrm{SiO}$ abundance is reached over similar timescales. It may be seen that the highest fractional abundances of $\mathrm{SiO}$ are attained for the lowest preshock density, $n_{\mathrm{H}}=10^{4} \mathrm{~cm}^{-3}$. At higher densities, both $\mathrm{O}_{2}$ and $\mathrm{OH}$, which are reactants in (1) and (2), are destroyed by the atomic hydrogen which is produced in the shock wave. Thus, the conversion of $\mathrm{Si}$ into $\mathrm{SiO}$ becomes incomplete at high density, and the gas-phase $\mathrm{SiO}$ abundance depends on $n_{\mathrm{H}}$ even though the Si sputtered fraction does not (cf. Fig. 2).

\subsection{Dependence on the transverse magnetic field strength}

The existence of a magnetic field transverse to the direction of propagation is a necessary condition for a C-type shock wave to form, and it is instructive to consider the variation of the structure of the shock wave with the strength of the magnetic field. Energy equipartition arguments, applied to the magnetic and thermal energy densities in the preshock molecular gas, of particle density $n\left(\mathrm{H}_{2}\right)+n(\mathrm{He})=0.6 n_{\mathrm{H}}$ and kinetic temperature $T$, suggest that $B^{2} /(8 \pi) \approx n_{\mathrm{H}} k_{\mathrm{B}} T$ and hence that $B=b n_{\mathrm{H}}^{0.5}$, where $b$ is a scaling parameter (cf. Sect. 3.2) such that $B$ is in $\mu \mathrm{G}$ when $n_{\mathrm{H}}$ is in $\mathrm{cm}^{-3}$; this is the proportionality adopted in the grid of models presented in Sect. 3.2. In gas of $T=10 \mathrm{~K}$, 

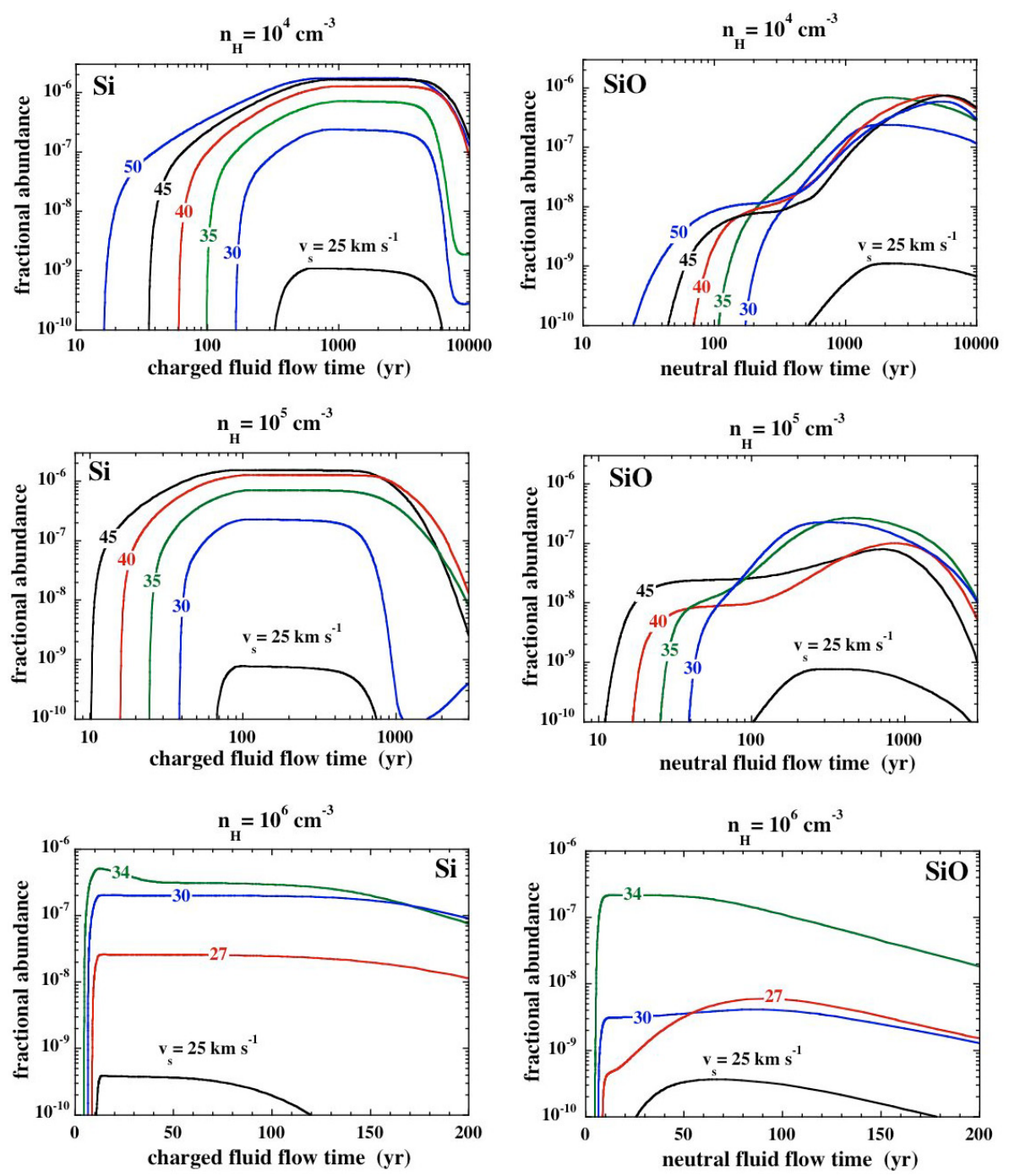

Fig. 3. The fractional abundances of $\mathrm{Si}$, released into the gas phase by the sputtering of olivine $\left(\mathrm{MgFeSiO}_{4}\right)$, and of $\mathrm{SiO}$, which subsequently forms in reactions (1) and (2). In the left-hand panels, the independent variable (abscissa) is the flow time of the charged fluid: see text, Sect. 3.2. equipartition with the thermal energy implies $b=0.18$. However, we note that such a low value of $b$ is inconsistent with the existence of a steady-state C-type shock wave when $n_{\mathrm{H}}=10^{5} \mathrm{~cm}^{-3}$ and $v_{\mathrm{s}} \geq 10 \mathrm{~km} \mathrm{~s}^{-1}$, as the corresponding ion magnetosonic speed in the preshock gas $\left(9.7 \mathrm{~km} \mathrm{~s}^{-1}\right)$ is lower than the shock speed.

In Fig. 5, we present results as a function of the transverse magnetic field strength, for the parameters of the model of Sch97: $n_{\mathrm{H}}=10^{5} \mathrm{~cm}^{-3}, v_{\mathrm{s}}=30 \mathrm{~km} \mathrm{~s}^{-1}$, and a magnetic field scaling parameter $b$ in the range $0.5 \leq b \leq 5$. We recall that Sch97 adopted $B=200 \mu \mathrm{G}$, corresponding to $b=0.63$. This value of $b$ is consistent with the analysis of Zeeman measurements by Crutcher (1999), who concluded that there was an approximate equipartition of the magnetic and kinetic energy densities in the molecular clouds that he had observed. It may be seen from Fig. 5 that increasing the magnetic field inhibits the release of Si from refractory grain cores in the shock wave, owing to the reduction in the maximum ion-neutral velocity difference, $\Delta v$. In Sect. 4.5, we consider how the magnetic field strength affects the relative intensities of the rotational transitions of $\mathrm{SiO}$.

\section{SiO rotational emission lines}

The observable quantities are the intensities of the rotational transitions of $\mathrm{SiO}$ and the velocity-profiles of these emission lines. Having computed the shock structure, we evaluate the line intensities and profiles as described in Appendix A, assuming that the shock is viewed face-on.

\subsection{Physical conditions in the SiO emission region}

Figure 6 illustrates the variation of physical conditions throughout the formation region of the $\mathrm{SiO} 5-4$ rotational line for our reference model with $n_{\mathrm{H}}=10^{5} \mathrm{~cm}^{-3}, v_{\mathrm{s}}=30 \mathrm{~km} \mathrm{~s}^{-1}$, and $b=0.63$. It may be seen that the line is optically thin through most of the hot precursor (where the flow speeds of the charged and neutral fluids differ), due to both the low $\mathrm{SiO}$ abundance and the large velocity gradient there. Therefore the line intensity is low despite a high kinetic temperature. At the rear of the shock wave, approaching maximum compression, the synthesis of $\mathrm{SiO}$ and the steady decrease in velocity gradient eventually raise the optical depth in the line, and the 5-4 intensity peaks, with the line temperature attaining values close to the local kinetic temperature of the neutral fluid, $T_{\mathrm{n}}$; that is, the line approaches LTE. The intensity then declines rapidly as the gas cools; the decline occurs in $500 \mathrm{yr}$ for the model shown here. This behaviour is insensitive to the rate of re-adsorption of $\mathrm{SiO}$ on to the grains, which occurs over much longer timescales.

In order to illustrate the dependence of conditions in the $\mathrm{SiO}$ emission region on the shock parameters, we present, in Fig. 7, the important physical quantities, evaluated at the peak of the SiO 5-4 line, for all models in our grid. We have verified 

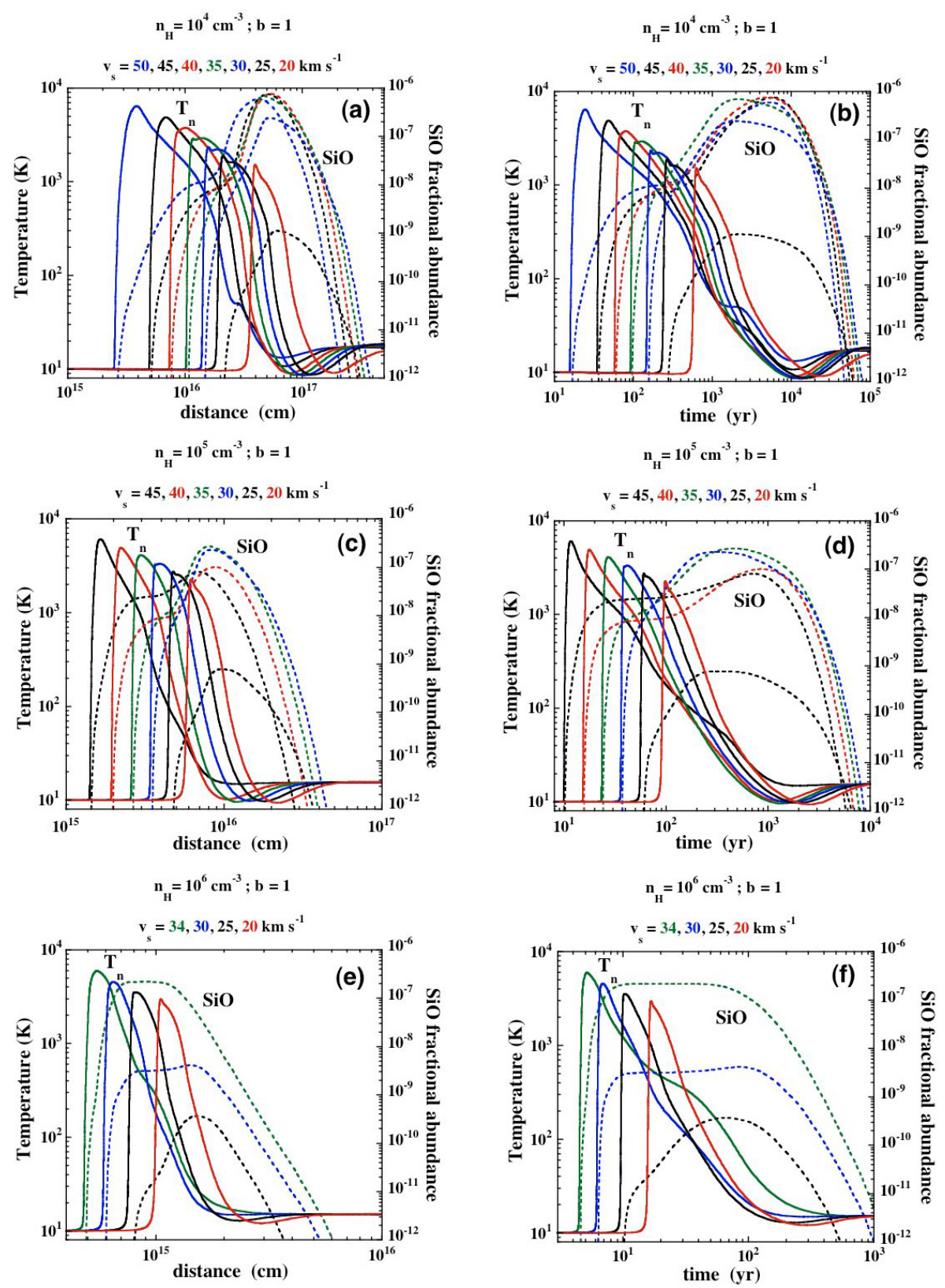

Fig. 4. The fractional abundance of $\mathrm{SiO}$, $n(\mathrm{SiO}) / n_{\mathrm{H}}$, computed for the grid of shock models and plotted as a function of distance (left) and neutral flow time (right); $n(\mathrm{SiO}) / n_{\mathrm{H}}$ is negligible for $v_{\mathrm{s}} \lesssim 20 \mathrm{~km} \mathrm{~s}^{-1}$. In addition, the temperature of the neutral fluid is plotted. (See also Fig. C.1 of Appendix C.)

that this is equivalent to computing intensity-weighted geometric means of the same quantities over the region where the 5-4 line intensity is more than $50 \%$ of its maximum value ${ }^{1}$. Hence, it provides a good indication of the mean characteristics of the region producing the peak of the emission.

Figure 7 shows that the $\mathrm{SiO}$ emission peak always occurs in the cool and dense postshock region: $T_{\mathrm{n}} \approx 50 \mathrm{~K}$ (Fig. 7a) and a density of 10-40 times the preshock value, close to maximum compression (Fig. 7b). The $\mathrm{SiO}$ fractional abundance, $n(\mathrm{SiO}) / n_{\mathrm{H}}$, is also approximately equal to its maximum value in the shock wave (compare Figs. 7c with 4). The trend to lower $\mathrm{SiO}$ abundance at higher $n_{\mathrm{H}}$, noted in Sect. 3.2, is clearly visible here. Finally, the "LVG parameter", $n(\mathrm{SiO}) /\left(\mathrm{d} v_{z} / \mathrm{d} z\right)$, lies typically in the range $10^{14}-10^{16} \mathrm{~cm}^{-2} \mathrm{~km}^{-1} \mathrm{~s}$, implying that the 5-4 line is optically thick at its peak for most models of our grid. In Sect. 4.5, we compare these physical parameters to values

\footnotetext{
1 Values evaluated at the peak also differ by less than a factor 2 from the same parameters evaluated at the "median" point where the integrated line intensity, $T \mathrm{~d} V(5-4)$, reaches half of its total.
}

inferred previously from LVG analyses of observations, assuming a uniform slab which fills the beam.

\subsection{Line profiles and peak line temperatures}

In Fig. 8, we compare the intensity profiles of various rotational lines, as functions of the flow speed of the neutral fluid, expressed in the frame of the preshock gas, for our reference model: $n_{\mathrm{H}}=10^{5} \mathrm{~cm}^{-3}, v_{\mathrm{s}}=30 \mathrm{~km} \mathrm{~s}^{-1}$, and $b=0.63$. The profiles are seen to be narrow (widths of $1-2 \mathrm{~km} \mathrm{~s}^{-1}$ ), with similar shapes and peaking within $2 \mathrm{~km} \mathrm{~s}^{-1}$ of $v_{\mathrm{s}}$, as expected for compressed material at the rear of the shock wave ${ }^{2}$. Note that

\footnotetext{
2 The maximum compression of the postshock relative to the preshock gas, $\sqrt{2} v_{\mathrm{s}} / v_{\mathrm{A}}$, where $v_{\mathrm{s}}$ is the shock speed and $v_{\mathrm{A}}$ is the Alfvén speed in the preshock gas, occurs when the magnetic pressure in the postshock gas is equal to the initial ram pressure. It follows that the flow speed in the postshock gas cannot fall below $v_{\min }=v_{\mathrm{A}} / \sqrt{2}=1.3 b \mathrm{~km} \mathrm{~s}^{-1}$ in the shock frame, where $b$ is the scaling parameter of the magnetic field, defined in Sect. 3.2.
} 

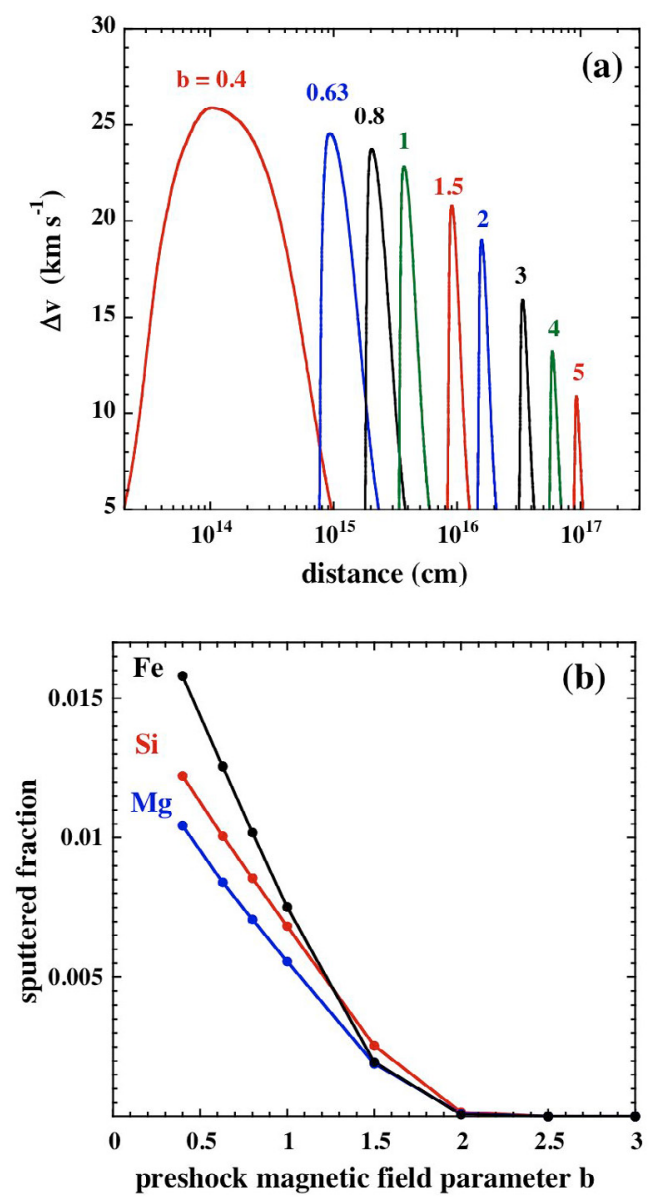

Fig. 5. a) The ion-neutral velocity difference, $\Delta v=\left|v_{\mathrm{i}}-v_{\mathrm{n}}\right|$, and $\left.\mathbf{b}\right)$ the fractions of $\mathrm{Mg}, \mathrm{Si}$ and $\mathrm{Fe}$ eroded from olivine $\left(\mathrm{MgFeSiO}_{4}\right)$ grains, computed as functions of the transverse magnetic field strength, $B$, in the preshock gas; $n_{\mathrm{H}}=10^{5} \mathrm{~cm}^{-3}$ and $v_{\mathrm{s}}=30 \mathrm{~km} \mathrm{~s}^{-1}$. Note that $B=b n_{\mathrm{H}}^{0.5}$, where $b$ is a scaling parameter (cf. Sect. 3.2) such that $B$ is in $\mu \mathrm{G}$ when $n_{\mathrm{H}}$ is in $\mathrm{cm}^{-3}$.

the transition 2-1 peaks further into the cooling flow, because the lower $j$-levels are repopulated from the higher levels as the temperature falls.

The general shape and centroid velocities are globally similar to those found by Sch97 (their Fig. 3b where the profiles were plotted in the shock frame), although the differences between the various lines are less significant in the present calculations. Also, the emission wing from the fast precursor, at the start of the shock wave, is weaker in the current models, owing to the delay in $\mathrm{SiO}$ formation (see Sect. 3.1), making our line profiles narrower than in Sch97. Note that including local thermal broadening in our profile calculations would not significantly change our predicted $\mathrm{SiO}$ line width of $1-2 \mathrm{~km} \mathrm{~s}^{-1}$, because the $\mathrm{SiO}$ emission peaks at low temperatures, $T_{n} \lesssim 100 \mathrm{~K}$, where the Doppler width is $\sqrt{k T / 44 m_{\mathrm{H}}} \leq 0.1 \mathrm{~km} \mathrm{~s}^{-1}$.

In the left column of Fig. 9, we show the predicted peak temperature of the SiO 5-4 line for the grid of models considered in Sect. 3.2, as well as the variation with $j_{\text {up }}$ of the peak brightness temperatures of various lines, relative to that of 5-4. The relative intensities have the advantage of being independent of the beam filling factor, and thus they are comparable directly to observations, without prior knowledge of the source size.

The relative peak intensities are within $20 \%$ of unity for $j_{\text {up }} \leq 7$ over a broad range of model parameters $\left(v_{\mathrm{s}} \geq 30 \mathrm{~km} \mathrm{~s}^{-1}\right.$ and $n_{\mathrm{H}}<10^{6} \mathrm{~cm}^{-3}$ ), owing to the large opacity and near-LTE
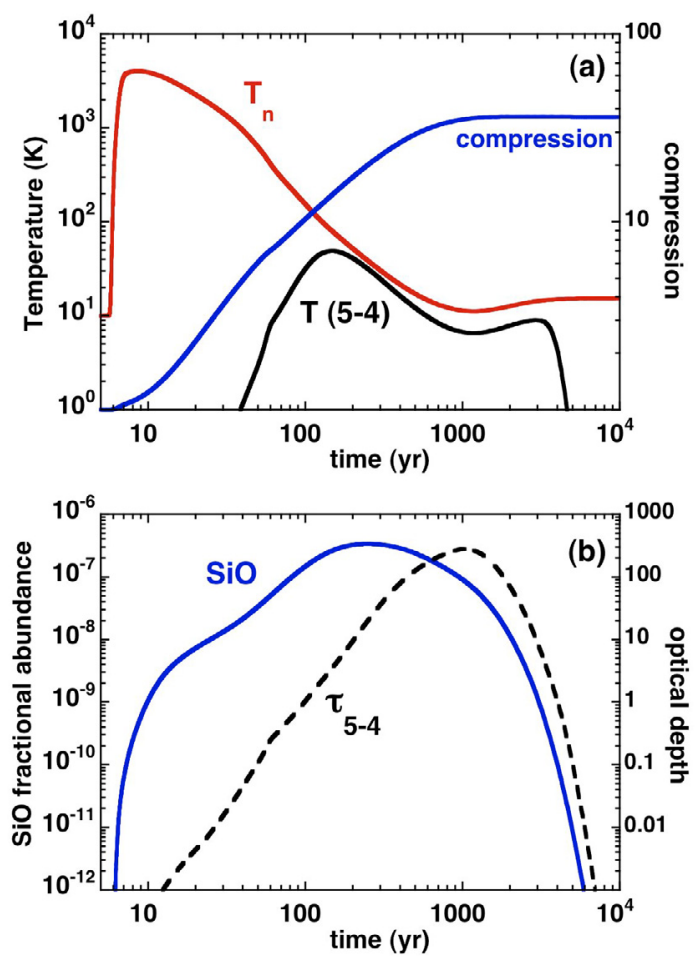

Fig. 6. a) The temperature of the neutral fluid, $T_{\mathrm{n}}$, the brightness temperature, $T(5-4)$, in the $j=5-4$ line, and the compression factor, $n_{\mathrm{H}} / n_{\mathrm{H}}$ (initial); b) the optical depth, $\tau_{5-4}$ in the 5-4 transition and the fractional abundance of $\mathrm{SiO}, n(\mathrm{SiO}) / n_{\mathrm{H}}$, as functions of the flow time of the neutral fluid, $t_{\mathrm{n}}$. The model parameters are $n_{\mathrm{H}}=10^{5} \mathrm{~cm}^{-3}$, $v_{\mathrm{s}}=30 \mathrm{~km} \mathrm{~s}^{-1}$, and $b=0.63$.

excitation conditions. For larger values of $j_{\text {up }}$, the relative intensities are more dependent on the shock speed and $\approx 1$ only when the limiting speed is approached. The absolute peak brightness temperature in the 5-4 line is typically $10-50 \mathrm{~K}$ for $v_{\mathrm{s}} \geq$ $30 \mathrm{~km} \mathrm{~s}^{-1}$, similar to the kinetic temperature in the emission region, but drops sharply at lower shock speeds, for which the $\mathrm{SiO}$ abundance (and opacity) is small. The broken curves in Figs. $9 \mathrm{~d}$ and $\mathrm{h}$ are the results obtained assuming that the initial abundance of $\mathrm{O}_{2}$ ice is negligible, i.e. the second of the two scenarios described in Sect. 3.1.

\subsection{Integrated line intensities}

In the right column of Fig. 9 are presented the integrated intensities (denoted $T \mathrm{~d} V$ ) of the rotational emission lines of $\mathrm{SiO}$, relative to the 5-4 line, computed for the grid of models considered in Sect. 3.2. There are significant differences between the relative integrated and peak $\left(T_{\text {peak }}\right)$ line temperatures (right and left columns, respectively, of Fig. 9), owing to systematic variations in linewidth with $j_{\text {up }}$, i.e. in the extent of the region where the line is significantly excited. The relative integrated intensities of lines with $j_{\text {up }} \geq 7$ remain the most sensitive to the shock speed. As may be seen in Sch97, the maximum value of $T \mathrm{~d} V$ occurs at higher values of the rotational quantum number, $j_{\text {up }}$, for higher $n_{\mathrm{H}}$. Although the shock temperature varies only weakly with $n_{\mathrm{H}}$ (see Fig. 4), a higher density enhances the rates of collisional excitation of the high- $j$ levels, for any given value of the shock speed, $v_{\mathrm{s}}$. In Sect. 5, we explore the usefulness of this effect for constraining the preshock density, based on a comparison with actual observations. 

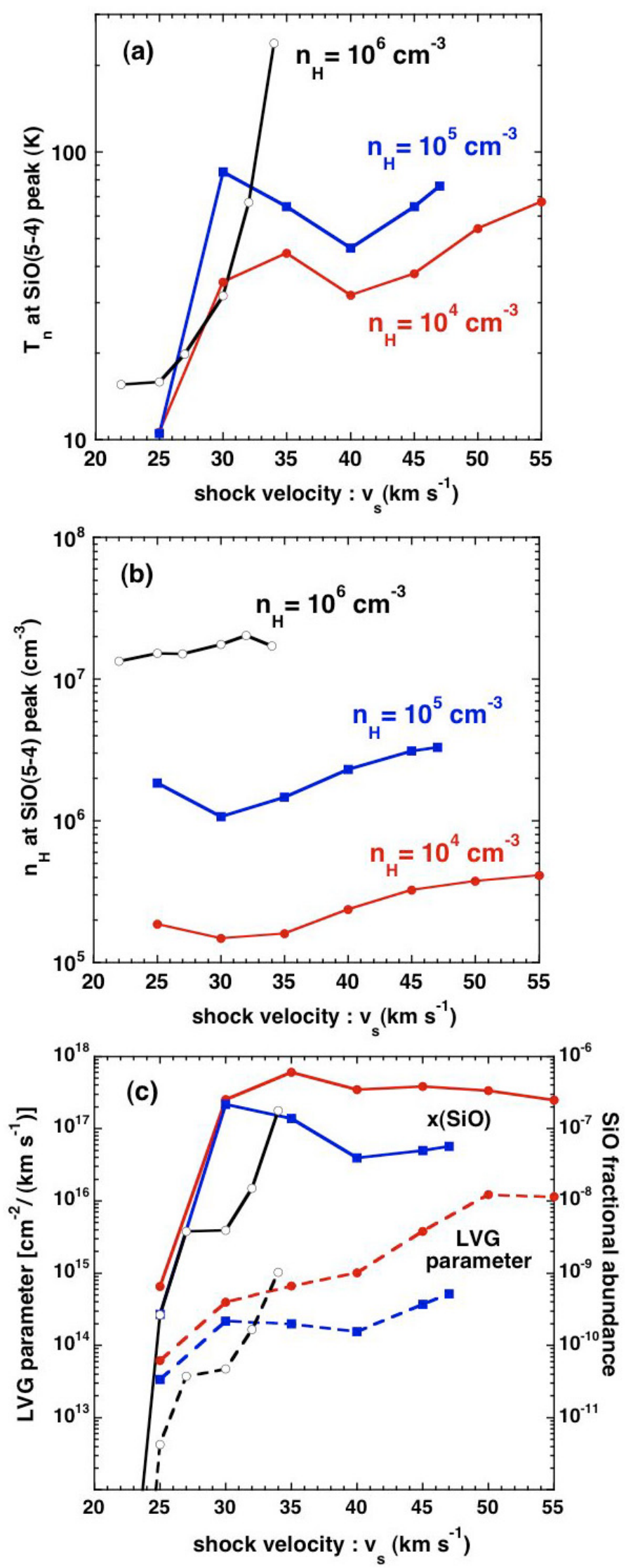

Fig. 7. Physical conditions at the position of the peak in the SiO 5-4 line intensity $\left[T_{\text {peak }}(5-4)\right]$ as functions of the shock speed, $v_{\mathrm{s}}$, for all models of the grid: a) neutral temperature, $T_{\mathrm{n}}$; b) preshock density, $n_{\mathrm{H}}$; c) the LVG parameter, $n(\mathrm{SiO}) /\left(\mathrm{d} v_{z} / \mathrm{d} z\right)$, and the fractional abundance of $\mathrm{SiO}$, $x(\mathrm{SiO}) \equiv n(\mathrm{SiO}) / n_{\mathrm{H}}$. (See also Fig. C.2 of Appendix C.)

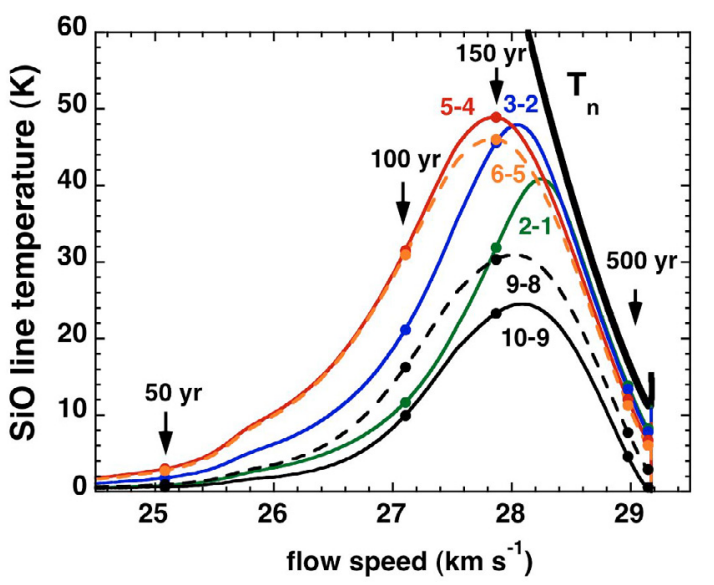

Fig. 8. The velocity profiles of transitions from rotational levels $j \rightarrow$ $j-1$ of $\mathrm{SiO}$, computed for our reference model, viewed face-on. Only those lines detectable from the ground are shown. The model parameters are $n_{\mathrm{H}}=10^{5} \mathrm{~cm}^{-3}, B=200 \mu \mathrm{G}$, and $v_{\mathrm{s}}=30 \mathrm{~km} \mathrm{~s}^{-1}$. The flow speed is in the reference frame of the preshock gas. The neutral temperature profile, $T_{\mathrm{n}}$, is shown also, as are indicative values of the flow time of the neutral fluid. (See also Fig. C.3 of Appendix C.)

The variation of the absolute integrated intensity of the 5-4 rotational emission line with the shock parameters is shown also in Fig. 9h. The bump in $T \mathrm{~d} V$ of the 5-4 transition, for $30 \leq v_{\mathrm{s}} \leq 40 \mathrm{~km} \mathrm{~s}^{-1}$ and $10^{4} \leq n_{\mathrm{H}} \leq 10^{5} \mathrm{~cm}^{-3}$ is caused by incomplete $\mathrm{O}_{2}$ destruction in the shock wave, resulting in more rapid $\mathrm{SiO}$ formation and warmer emission zones (cf. Fig. 7). As the broken curves in Figs. 9d and h show, this "bump" is absent in our second scenario, where $\mathrm{O}_{2}$ is never abundant in the gas phase. At higher shock speeds, the results from the two scenarios become identical, as $\mathrm{OH}$ dominates the oxidation of $\mathrm{Si}$ in both cases.

\subsection{Influence of viewing angle}

Statistically, there is a low probability that a planar shock should happen to be viewed face-on. Accordingly, we have explored the effects on the $\mathrm{SiO}$ rotational line intensities of varying the viewing angle, for the case of our reference model, using the formula (A.16), derived in Appendix A.

The variations of $T_{\text {peak }}(5-4)$ and $T \mathrm{~d} V(5-4)$ with viewing angle, $\theta$, are plotted in the bottom panel of Fig. 10. The peak intensity is almost unaffected by the inclination, as the line is already optically thick for a face-on view (see Eq. (A.16)). On the other hand, the velocity projection reduces the line width, and the integrated intensity, $T \mathrm{~d} V$, declines steadily with increasing viewing angle - by up to a factor of 2 at $75^{\circ}$. However, such a variation would be difficult to deduce from observations, given the typical uncertainties in beam filling factors.

Panels (a) and (b) of Fig. 10 illustrate the changes in the (relative to 5-4) peak and integrated $\mathrm{SiO}$ line temperatures. Significant changes, compared with viewing face-on, are seen only for inclinations greater than $60^{\circ}$ from the normal, and they affect only the optically thin lines $j_{\text {up }} \leq 3$ and $j_{\text {up }} \geq 12$. The main change in the curves for the relative integrated line temperatures (panel (b) of Fig. 10) is that the maximum occurs at lower $j_{\text {up }}$ as $\theta$ increases; this effect could be easily confused with a face-on shock of slightly lower preshock density (cf. Fig. 9). The relative peak temperature (panel (a)) is even more strongly modified, with an upward turn of the curve at $j_{\text {up }} \leq 4$. The latter characteristic appears to be the only unambiguous signature of a 

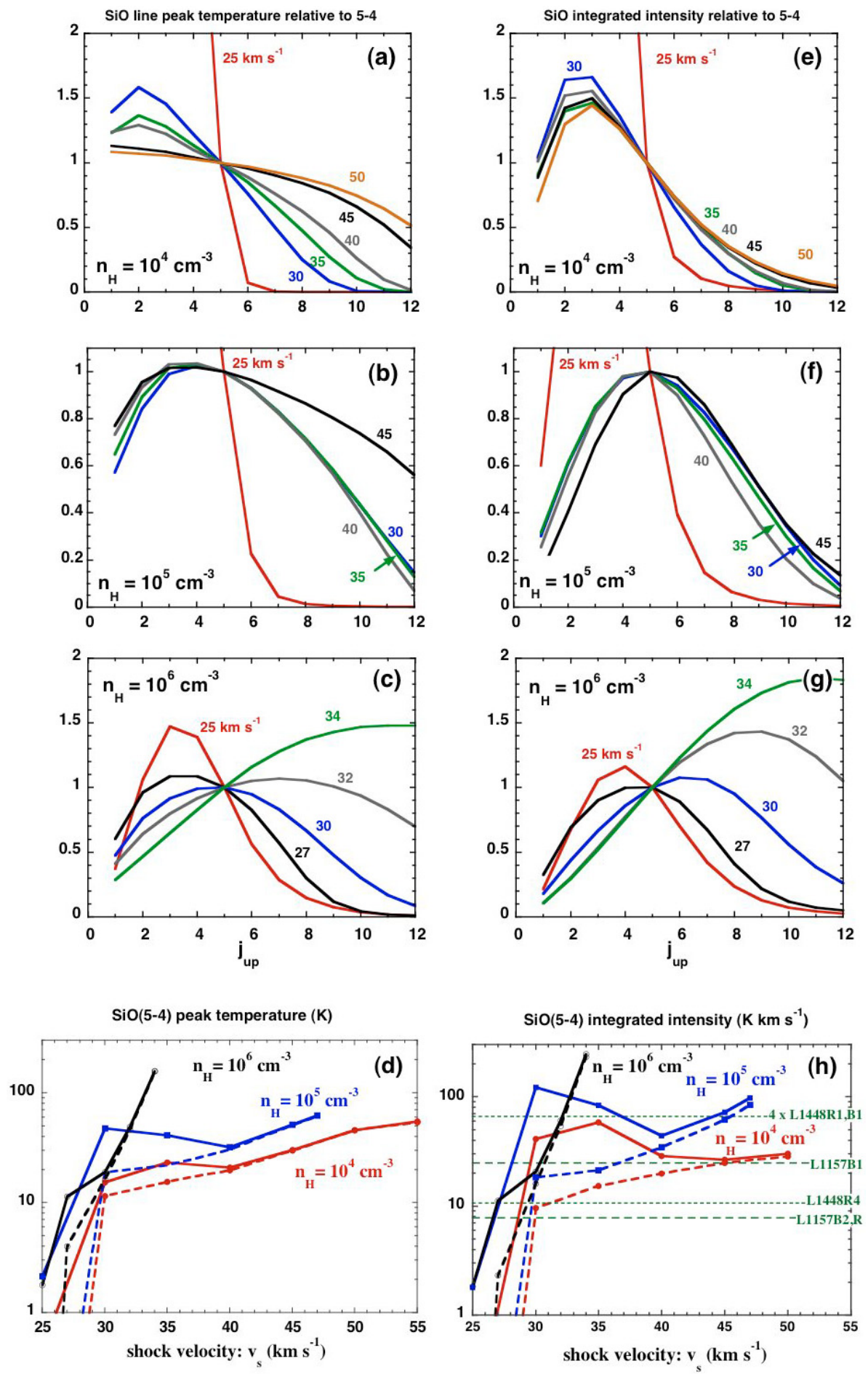

Fig. 9. a)-c) The peak line temperatures, $T_{\text {peak }}$, of the rotational emission lines of $\mathrm{SiO}$, relative to the 5-4 line, as functions of the rotational quantum number of the upper level of the transition, $j_{\text {up }}$, for the grid of models in Sect. 3.2. The value of density, $n_{\mathrm{H}}$, of the preshock gas is indicated in each panel. d) The absolute peak brightness temperature of the 5-4 line, $T_{\text {peak }}(5-4)$, as a function of shock speed, $v_{\mathrm{s}}$, for all three values of the preshock gas density, $n_{\mathrm{H}}$. Shock speeds in excess of $34 \mathrm{~km} \mathrm{~s}^{-1}$ are absent when $n_{\mathrm{H}}=10^{6} \mathrm{~cm}^{-3}$, as they give rise to a J-type discontinuity, and the shock wave is no longer C-type; see Sect. 3.2. The right-hand panels e)-h) show the corresponding values of the integrated line intensities, $T \mathrm{~d} V$. The values of $T \mathrm{~d} V(5-4)$ observed in L1157 and L1448 are indicated. In panels $\mathbf{d}$ ) and $\mathbf{h}$ ), the broken curves show the results obtained assuming that the initial abundance of $\mathrm{O}_{2}$ ice is negligible, i.e. the second of the two scenarios described in Sect. 3.1.

viewing angle $>60^{\circ}$ in the context of our one-dimensional models.

\subsection{Influence of the transverse magnetic field strength}

In Sect. 3.3, we have seen that the efficiency of sputtering $\mathrm{Si}$ from grains decreases monotonically with increasing magnetic field strength. The effect of varying the scaling parameter, $b$, on the emergent $\mathrm{SiO}$ line intensities is shown in Fig. 11, for our reference model.

In panels $\mathrm{a}$ and $\mathrm{b}$ of Fig. 11 are plotted the predicted line profiles, peak temperatures, and integrated intensities of the
SiO 5-4 line, for various values of $b$. It may be seen that the maximum intensity is reached for intermediate values of $0.63 \lesssim b \lesssim 1$. At smaller $b, \mathrm{SiO}$ is less abundant: the shock wave is narrower and hotter, and so $\mathrm{O}_{2}$ is more readily destroyed by $\mathrm{H}$, resulting in incomplete oxidation of $\mathrm{Si}$ into $\mathrm{SiO}$. At larger $b$, the $\mathrm{SiO}$ emission decreases owing to less efficient sputtering of Si (see Fig. 5b) at the lower ion-neutral drift speeds. In particular, the predicted intensity drops from $T_{\text {peak }}=10 \mathrm{~K}$ at $b=2$ to practically zero at $b=3$.

Panels $\mathrm{c}$ and d of Fig. 11 show the relative peak and integrated line temperatures, as functions of $j_{\text {up }}$, for various values of $b \leq 2$ (curves for $b>2$ are not shown as they lead to negligible $\mathrm{SiO}$ emission). It may be seen that the values $0.63 \lesssim b \lesssim 1$, 

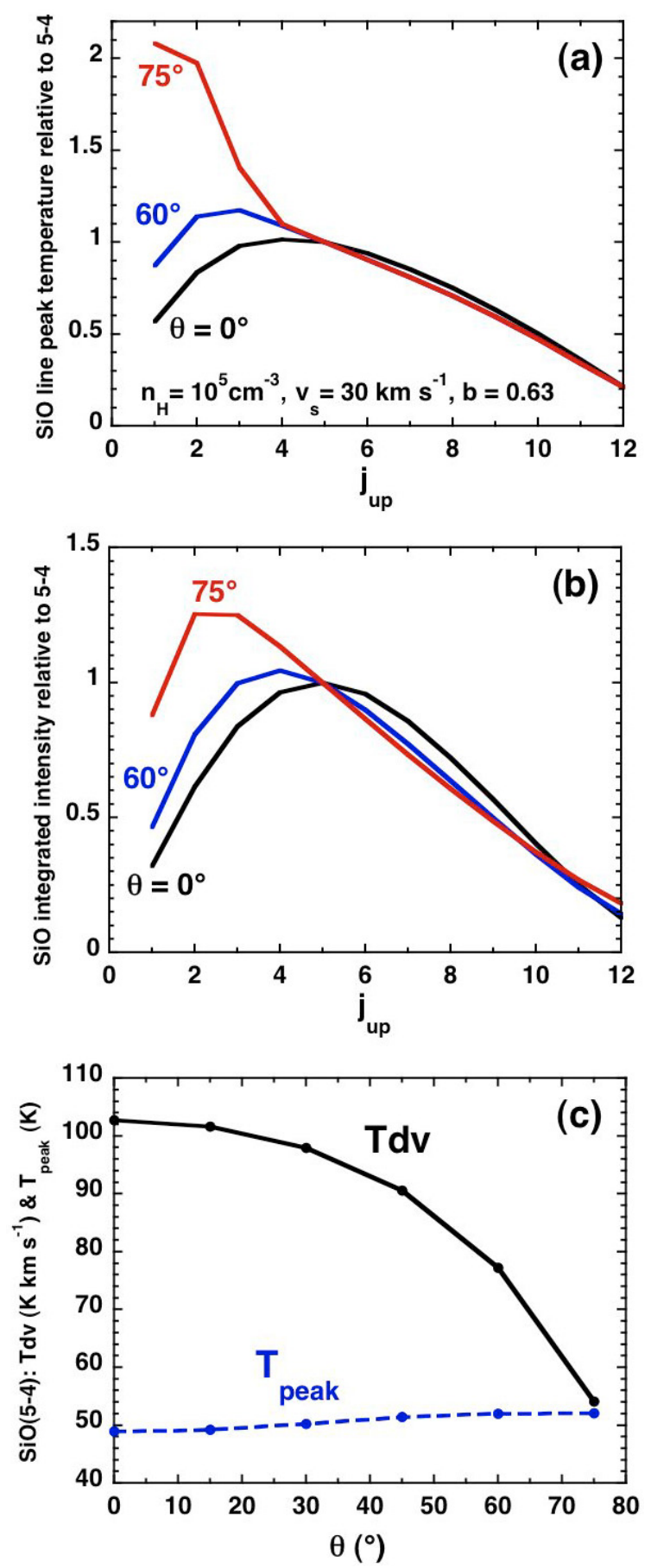

Fig. 10. Effect of the inclination angle, $\theta$, on a) the peak, and b) the integrated intensities of the rotational emission lines of $\mathrm{SiO}$, relative to the 5-4 line; c) the integrated and peak intensities of the 5-4 line.

which give rise to the strongest 5-4 emission, also yield the highest relative intensities of lines from $j_{\text {up }} \geq 7$. For example, the intensity of the $11-10$ line, relative to $5-4$, is 3 to 4 times larger than when $b=0.5$ or $b=1.5$. Comparison with Fig. 9 suggests that this dependence on $b$ may be difficult to distinguish observationally from variations in shock speed. A less ambiguous indication of the value of $b$ might be obtained from the width of the cooling zone, which increases as $b^{2}$, from $10^{15} \mathrm{~cm}$ for $b=0.4$ to $2 \times 10^{16} \mathrm{~cm}$ for $b=2$ and for the parameters of our reference model (see Fig. 5a).

\section{Comparisons with observations}

\subsection{Rotational line profiles}

As Sch97 first noted, the generic SiO line profile predicted by steady planar C-type shock waves, with a peak at high velocity (in the postshock gas) and a tail at lower velocity (in the accelerating precursor), is reminiscent of the $\mathrm{SiO}$ line profiles in the L1448 molecular jet (Bachiller et al. 1991). Similarly, we note that the reversed shape of $\mathrm{SiO}$ profiles in the L1157 bowshocks, with a peak at low velocity and a high velocity tail (Zhang et al. 1995), could arise if the postshock gas is stationary in the cloud frame, i.e. if one observes the reverse shock, in which the jet is being decelerated. However, in either case, the $\mathrm{SiO}$ profiles predicted by our models remain narrower than those observed, with widths of $0.5-2 \mathrm{~km} \mathrm{~s}^{-1}$, as compared to the observed widths of $5-20 \mathrm{~km} \mathrm{~s}^{-1}$ in $3^{\prime \prime}-10^{\prime \prime}$ beams.

Broader line profiles from steady C-type shocks could arise if $\mathrm{Si}$ was sputtered not only from grain cores, as assumed here, but also from $\mathrm{SiO}$-containing grain mantles, with lower binding energy. Then, the $\mathrm{SiO}$ abundance would be much enhanced at intermediate velocities, in the precursor (see Sch97). However, owing to the steep temperature decline across the shock wave, this situation results in large variations of the line widths and velocity centroids with the emitting rotational level, $j_{\text {up }}$ (cf. Fig. 5 of Sch97). In fact, the observed profiles are very similar from line to line, with the $(8-7) /(2-1)$ intensity ratio showing only modest variations with velocity (see, for example, Fig. 9 of Nisini et al. 2007). These observations suggest that the broad $\mathrm{SiO}$ lines are not attributable entirely to intrinsic velocity gradients through a single, planar C-type shock wave. There may be several shock-cooling zones inside the beam, each with a narrow intrinsic profile, which appear spread out in radial velocity owing to a range of inclination angles or propagation speeds, in the observer's frame. This conclusion is supported by interferometric observations of L1157 and L1448 (Guilloteau et al. 1992; Gueth et al. 1998; Benedettini et al. 2007), which reveal systematic velocity gradients across the $\mathrm{SiO}$ emitting knots (reminiscent, in some cases, of a bowshock geometry; Dutrey et al. 1997) down to $2^{\prime \prime}-3^{\prime \prime}$ resolution; at the distances of L1157 and L1448, the angular dimensions of the $\mathrm{SiO}$ emitting regions in Fig. 8, for example, are a few tenths of an arcsec. Such complex two-dimensional modelling lies outside the scope of the present paper. However, we argue in Sect. 5.3 that we may still perform a meaningful comparison of our predicted $\mathrm{SiO}$ line intensities with observations of knots in outflows, without reproducing in detail the line profiles, provided that the shock conditions do not vary too much across the beam.

\subsection{Narrow SiO lines near ambient velocity}

In addition to the typically broad $\mathrm{SiO}$ line profiles mentioned above, some outflow regions such as NGC 1333 and L1448 exhibit extremely narrow $\mathrm{SiO}$ emission lines, with $\Delta v \approx$ $0.5 \mathrm{~km} \mathrm{~s}^{-1}$, near rest velocity (Lefloch et al. 1998; Codella et al. 1999; Jiménez-Serra et al. 2004, 2005). The corresponding $\mathrm{SiO}$ abundance of $10^{-11}-10^{-10}$ is two to three orders of magnitude smaller than in the broad $\mathrm{SiO}$ components (Codella et al. 1999; Jiménez-Serra et al. 2005). Jiménez-Serra et al. proposed that this feature in L1448 traces a magnetic precursor, where neutral gas is just beginning to accelerate and grain species are starting to be released into the gas phase. However, as we now explain, detailed multifluid shock models do not support this interpretation. 

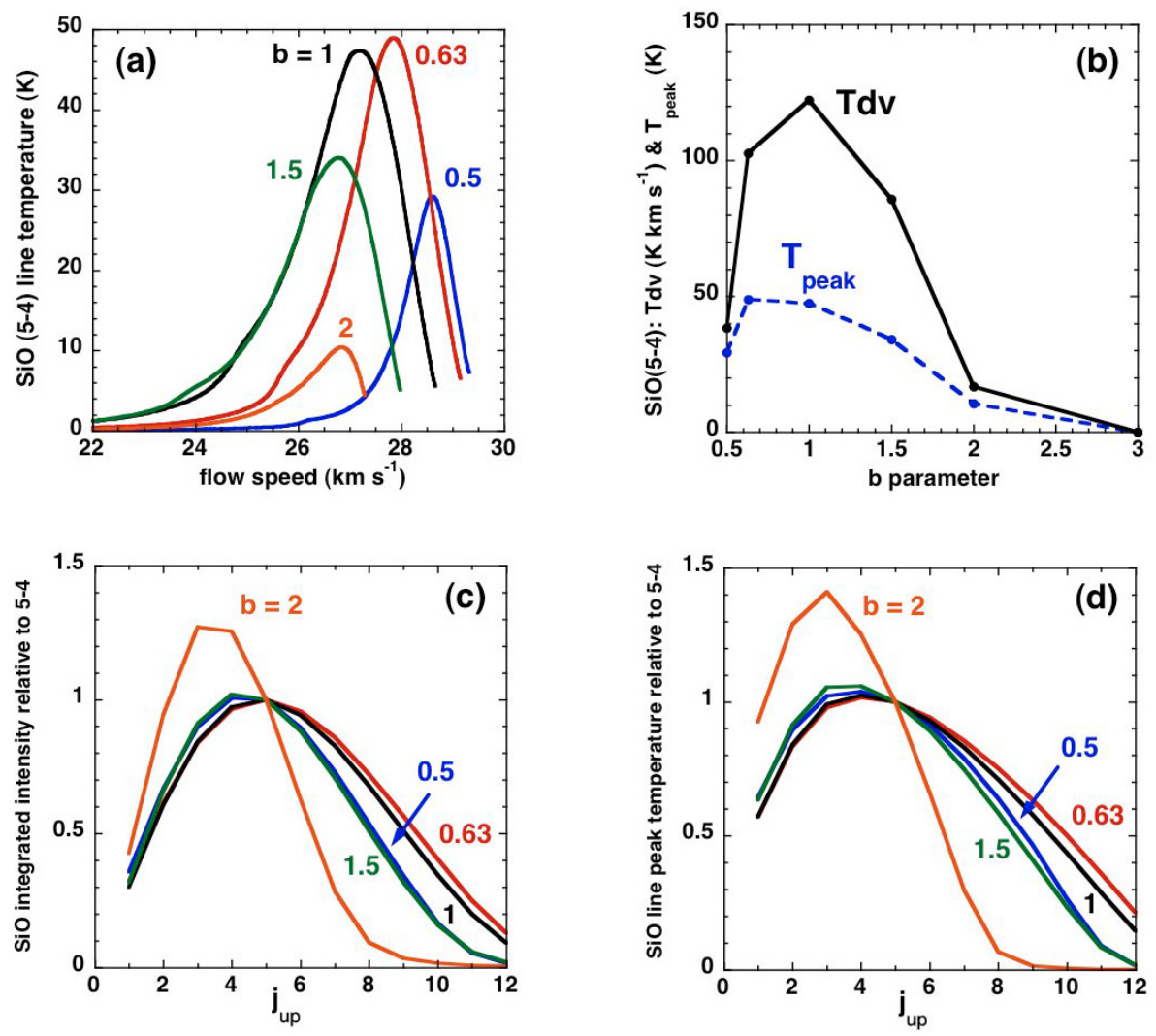

Fig. 11. a) The $\mathrm{SiO} 5-4$ line temperature, as a function of the flow speed of the neutral fluid, in the reference frame of the preshock gas, for the specified values of the magnetic field parameter, $b$; b) the peak and integrated intensities of the 5-4 line, as functions of $b$; c) the integrated and $\mathbf{d}$ ) the peak intensities of rotational emission lines $j_{\text {up }} \rightarrow j_{\text {up }}-1$ of $\mathrm{SiO}$, relative to the 5-4 transition, for the specified values of $b$. All calculations for $v_{\mathrm{s}}=30 \mathrm{~km} \mathrm{~s}^{-1}$ and $n_{\mathrm{H}}=10^{5} \mathrm{~cm}^{-3}$.

Our $\mathrm{SiO}$ line profile calculations show that emission from a magnetic precursor does not give rise to a narrow feature near the speed of the preshock gas. The line intensity increases as the neutral fluid is accelerated, heated, and enriched in $\mathrm{SiO}-$ by orders of magnitude by the time that grain-sputtering is complete. Indeed, this deduction could have been made already, on the basis of Figs. 3 and 5 of Sch97, which cover the entire velocity range relevant to predicting the $\mathrm{SiO}$ line profiles. Truncation of the precursor when the neutral fluid has been accelerated to only $v_{\mathrm{n}}=0.5 \mathrm{~km} \mathrm{~s}^{-1}$ would imply a very finely-tuned shock age, a circumstance which appears to us to be improbable. Furthermore, an ion-neutral drift speed of at least $5 \mathrm{~km} \mathrm{~s}^{-1}$ is needed to start releasing species from grain mantles, where binding energies are a few tenths of an eV (Flower \& Pineau des Forêts 1994), and of at least $20 \mathrm{~km} \mathrm{~s}^{-1}$ to start sputtering grain cores (May et al. 2000). However, the $\mathrm{H}^{13} \mathrm{CO}^{+}$line does not show evidence of this predicted acceleration: its emission peak is shifted by only $+0.5 \mathrm{~km} \mathrm{~s}^{-1}$ from the velocity of the ambient gas, like the narrow $\mathrm{SiO}$ feature (Jiménez-Serra et al. 2004).

We believe that a more likely explanation of the narrow $\mathrm{SiO}$ feature in $\mathrm{L} 1448$ is that it traces Si-enriched postshock material that has been decelerated by and mixed with the ambient gas, as proposed originally by Lefloch et al. (1998) and Codella et al. (1999) in connection with other regions. Given a shock speed $v_{\mathrm{s}} \leq 30 \mathrm{~km} \mathrm{~s}^{-1}$ and the high ambient density characteristic of Class 0 protostellar envelopes, deceleration could be achieved readily within the L1448 flow age of approximately 3500 yr. The low SiO fractional abundance would then be a consequence of mixing with $\mathrm{SiO}-$ poor ambient gas. Alternatively, the narrow feature might arise in a reverse C-type shock, where outflow material at $v<20 \mathrm{~km} \mathrm{~s}^{-1}$ is brought almost to rest by the much denser ambient medium, and the shock speed is too low to produce abundant $\mathrm{SiO}$. Both interpretations are consistent with
$\mathrm{NH}_{3}$ observations of dense gas in the envelope of the L1448 protostar, with radial velocity and spatial extent similar to that of the narrow $\mathrm{SiO}$ feature and signs of heating near the path of the fast L1448 jet (Curiel et al. 1999).

\subsection{SiO line intensities}

If our explanation of $\mathrm{SiO}$ profile broadening is correct, one could in principle recover the parameters of each individual emission zone in the beam by analysing the relative intensity ratios as functions of velocity. Unfortunately, such data are currently quite noisy and not yet available for a wide range of values of $j_{\text {up }}$. Furthermore, knowledge of the beam-filling factor as a function of velocity would be necessary to obtain absolute intensities and remove ambiguity in the shock parameters; but this would require sub-arcsecond angular resolution, which is not yet available. Nevertheless, one may still derive some approximate beam-averaged shock properties, if all of the shock components have similar excitation conditions, as is suggested by single-dish data, which show the line ratios to be insensitive to velocity, $v$. In this case, the observed profile will be simply a convolution of the individual, narrow shock profiles with the (unknown) filling-factor, $\phi(v)$. The observed absolute $T \mathrm{~d} V$ is simply that for a single shock, multiplied by the total beam filling factor of the SiO-emitting region in the beam, $f=\int \phi(v) \mathrm{d} v$, as inferred from its overall size in single-dish maps. The values of $T \mathrm{~d} V$ for different $j_{\text {up }}$, relative to the 5-4 transition, remain unchanged compared to a single shock, because $f$ cancels out in the ratios, thereby enabling direct comparison with our models. In the following, we assume that this situation prevails.

By way of illustration of the applicability of the shock models, we show in Fig. 12 the relative integrated intensities of the 

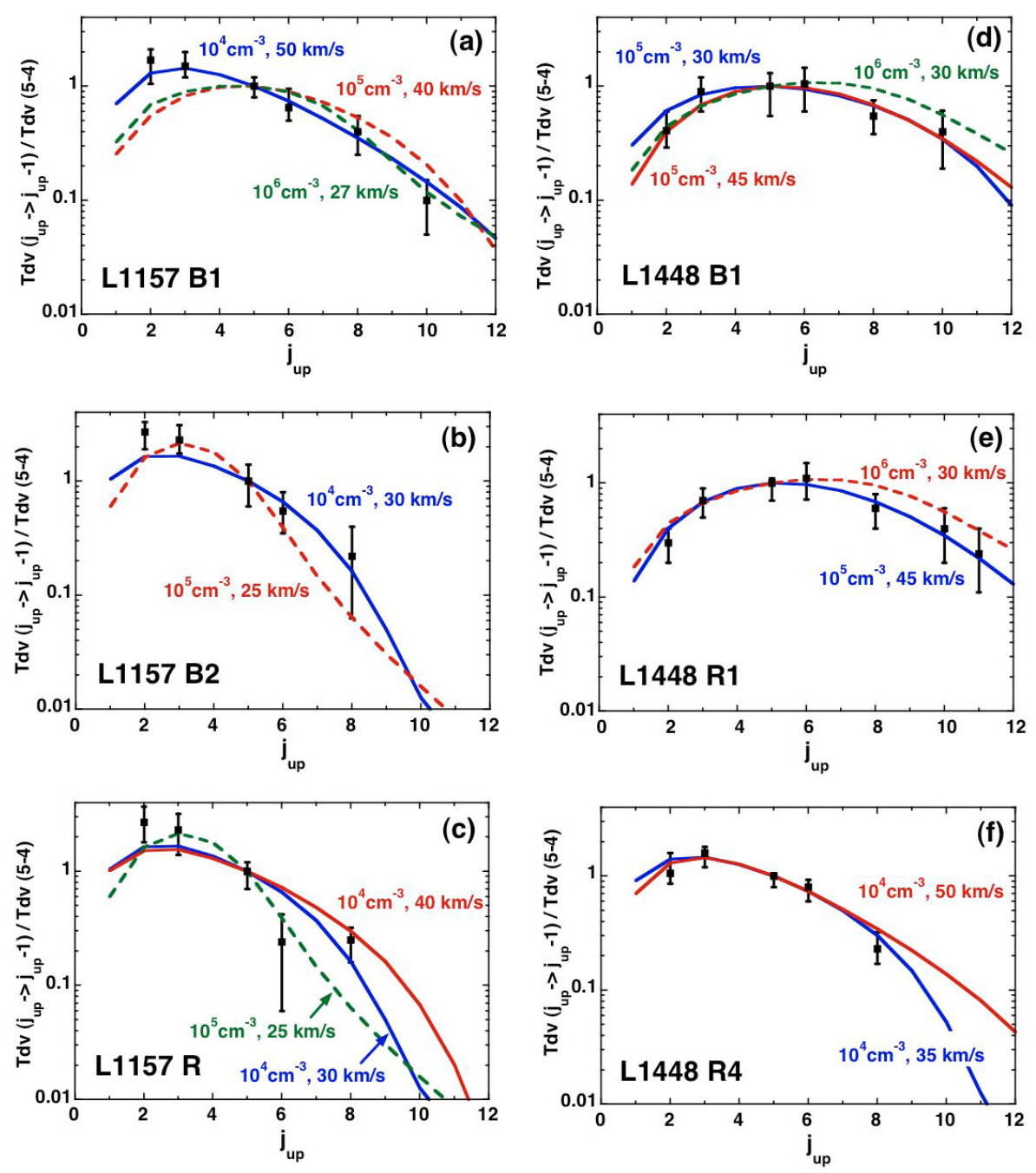

Fig. 12. The relative intensities of the rotational emission lines of $\mathrm{SiO}$ observed in the outflow sources L1157 and L1448 (Nisini et al. 2007: points with error bars) and predicted by the C-type shock models (curves) with the parameters $\left(n_{\mathrm{H}}, v_{\mathrm{s}}\right)$ indicated; see text, Sect. 5. The data points which are plotted include a correction for differing beam sizes, which is significant for low- $j$ lines. Full curves denote the bestfitting models of our grid. Broken curves show "near-miss" models with a different value of $n_{\mathrm{H}}$, which either yield a worse fit to the data points or do not reproduce the absolute $T \mathrm{~d} V$ of the 5-4 line. (See also Fig C.4 of Appendix C.) rotational transitions of $\mathrm{SiO}$ observed in the outflow sources L1157 and L1448 (Nisini et al. 2007) and predicted by the grid of models, whose parameters are specified; in all cases, the magnetic field scaling parameter $b=1$. As noted in Sect. 4.5, this value of $b$ yields the largest relative intensities of the high- $j$ lines and hence will yield a lower limit to the shock speed required to reproduce the observations (except at $n_{\mathrm{H}}=10^{5} \mathrm{~cm}^{-3}$, where high- $j$ excitation is a non-monotonic function of $v_{\mathrm{s}}$; see Fig. 9f). We assume also that the shock wave is viewed face-on; if the true inclination exceeds $60^{\circ}$, this assumption results in the preshock density being slightly underestimated (see Sect. 4.4). The models shown as the full curves are those which provide the best fits to the observations. In order to illustrate how well the shock parameters are constrained, we plot as dashed curves "near-miss" models that fit most of the data points, or fit all points but do not reproduce the absolute intensity of the 5-4 line (see below).

Figure 12 demonstrates that steady-state C-type shocks with $\mathrm{Si}$-sputtering from grain cores can reproduce successfully the relative integrated intensities of $\mathrm{SiO}$ lines in these molecular outflows. Furthermore, Fig. 9h shows that the models can reproduce also the absolute integrated intensity of $\mathrm{SiO} 5-4$ with the estimated beam filling factors $f \approx 1$ in L1157 and L1448-R4 and $f \simeq 1 / 4$ in L1448 R1 and B1 (cf. Nisini et al. 2007). Alternative fits with higher density and lower shock speeds $\left(n_{\mathrm{H}}=10^{6} \mathrm{~cm}^{-3}\right.$ and $27 \lesssim v_{\mathrm{s}} \lesssim 30 \mathrm{~km} \mathrm{~s}^{-1} ; n_{\mathrm{H}}=10^{5} \mathrm{~cm}^{-3}$ and $\left.v_{\mathrm{s}}=25 \mathrm{~km} \mathrm{~s}^{-1}\right)$ underestimate $T \mathrm{~d} V(5-4)$ and are thus ruled out. The shock speed, $v_{\mathrm{s}}$, is constrained to within $15 \mathrm{~km} \mathrm{~s}^{-1}$ and the preshock density to within a factor of 10 , with one of our grid values of $n_{\mathrm{H}}$ yielding a clear best fit in all cases.

In Appendix C, we present, in Fig. C.4 results equivalent to those in Fig. 12, but for the secondary grid of models, in which oxygen is initially in the form of $\mathrm{H}_{2} \mathrm{O}$ ice rather than $\mathrm{O}_{2}$ ice. Again, the relative intensities can be well reproduced by steady-state C-type shocks. The absolute $\mathrm{SiO} T \mathrm{~d} V(5-4)$ favour the low $n_{\mathrm{H}}$, high $v_{\mathrm{S}}$ cases (cf. the dashed curved in Fig. 9h). The best-fit shock parameters and the inferred physical conditions at the (5-4) line peak are almost unchanged, as the range of shock speeds is such that oxidation of $\mathrm{Si}$ by $\mathrm{OH}$ is dominant.

It is instructive to compare the physical parameters at the $\mathrm{SiO}$ peak of our best-fit, steady-state C-type shock models to those previously inferred from an LVG analysis, assuming a slab of constant density, temperature, and velocity gradient along the line of sight (Nisini et al. 2007). From Table 1, it may be seen that the "slab LVG" approach yields similar values of the density to our shock models but overestimates by a factor 5-10 the kinetic temperature and underestimates by several orders of magnitude the Sobolev LVG opacity parameter. The cool postshock layer emits over a narrow velocity range and needs to be more optically thick in $\mathrm{SiO}$ to produce the same $T \mathrm{~d} V$ as a hot slab with a large velocity gradient along the line of sight. On the other hand, similar $\mathrm{SiO}$ abundances are deduced using both approaches, to within typically a factor of 3 .

We note that the models should be able to simulate also the other spectral observations of the sources, notably the $\mathrm{H}_{2}$ line 
Table 1. Properties of SiO-emission regions deduced from face-on C-type shock models or homogeneous-slab LVG models.

\begin{tabular}{l|llllll|llll}
\hline \hline & \multicolumn{5}{|c|}{ Face-on C-type shock models ${ }^{a, b}$} & \multicolumn{4}{c}{ Slab LVG models $^{a, c}$} \\
SiO knot & $n_{\mathrm{H}}^{\text {init }}$ & $v_{\mathrm{s}}$ & $T_{\text {kin }}$ & $n_{\mathrm{H}}$ & LVG & $x_{\mathrm{SiO}}$ & $T_{\text {kin }}$ & $n_{\mathrm{H}}$ & LVG & $x_{\mathrm{SiO}}$ \\
\hline L1448 B1 & $1(5)$ & 30,45 & $90-70$ & $10-30$ & $2-4$ & $0.5-2$ & $>500$ & 8 & 0.1 & 1 \\
L1448 R1 & $1(5)$ & 45 & 70 & 30 & 4 & 0.5 & $>500$ & 10 & 0.1 & 1 \\
L1448 R4 & $1(4)$ & $35-50$ & $45-55$ & $1.5-4$ & $7-100$ & $3-7$ & 200 & 2.5 & 0.03 & 0.3 \\
\hline L1157 B1 & $1(4)$ & 50 & 55 & 4 & 100 & 3 & $150-300$ & 3 & 0.08 & 0.8 \\
L1157 B2 & $1(4)$ & 30 & 35 & 1.5 & 4 & 2 & $200-300$ & 2 & 0.05 & \\
L1157 R & $1(4)$ & $30-40$ & $35-30$ & $1.5-2$ & $4-10$ & $2-3$ & $50-100$ & $1-5$ & 0.02 & 0.6 \\
\hline
\end{tabular}

${ }^{a}$ Units: $n_{\mathrm{H}}^{\text {init }}$ is the preshock density, in $\mathrm{cm}^{-3} ; v_{\mathrm{S}}$ the shock speed, in $\mathrm{km} \mathrm{s}^{-1} ; T_{\text {kin }}$ is the local gas kinetic temperature, in $\mathrm{K} ; n_{\mathrm{H}}$ is the local gas density, in $10^{5} \mathrm{~cm}^{-3}$; "LVG" is the local LVG parameter, $n(\mathrm{SiO}) /\left(\mathrm{d} v_{\mathrm{n}} / \mathrm{d} z\right)$, in $10^{14} \mathrm{~cm}^{-2} \mathrm{~km}^{-1} \mathrm{~s}$; and $x_{\mathrm{SiO}}$ is the local fractional abundance, in units of $10^{-7}$.

${ }^{b}$ Best grid model from Fig. 12 and physical parameters at the SiO 5-4 line peak from Fig. 7.

${ }^{c}$ Values taken from Tables 4 and 5 of Nisini et al. (2007). The LVG parameter is given by $N(\mathrm{SiO}) / \Delta V$, with $\Delta V=10 \mathrm{~km} \mathrm{~s}^{-1}$.

intensities. In a forthcoming publication, we shall consider in detail the outflow L1157 and make a more comprehensive comparison of its observed spectrum with the predictions of shock models.

\section{Concluding remarks}

We have considered the structure of C-type shock waves propagating into molecular gas containing amorphous carbon and silicate grains; olivine $\left(\mathrm{MgFeSiO}_{4}\right)$ was chosen as the representative silicate-grain material. We find that the degree of sputtering of silicon from the grains is smaller, by about an order of magnitude, than was predicted by the calculations of Sch97, owing partly to higher sputtering thresholds and lower sputtering yields but principally to the reduced width of the shock wave in the present calculations. The reduction in the shock width is a consequence of a more accurate treatment of the coupling between the neutral fluid and the charged grains in the current model.

A grid of C-type shock models has been computed, for values of the preshock gas density and the shock speed which are believed to span the ranges of these parameters in molecular outflows; two scenarios were considered regarding the initial distribution of oxygen in the gas and solid phases. The maximum speed of a C-type shock is limited by the inertia of the (charged) grains in the preshock gas and by the collisional dissociation of molecular hydrogen within the shock wave, which can lead to a J-type discontinuity (Le Bourlot et al. 2002; Flower $\&$ Pineau des Forêts 2003). We find that significant sputtering of the grain cores occurs only for shock speeds $v_{\mathrm{s}} \geq 25 \mathrm{~km} \mathrm{~s}^{-1}$ and moderate magnetic field strengths close to equipartition with the cloud kinetic energy (magnetic field parameter $0.5 \leq b \leq 2$ ). However, we note that the sputtering threshold energy is determined principally by the so-called "displacement energy", $E_{\mathrm{D}}$, of the material, whose value for the silicates of relevance here remains uncertain (May et al. 2000). The sputtering yields increase rapidly from threshold, and a reduction in the threshold energy would enable significant sputtering to occur at lower shock speeds or higher magnetic field strengths.

We find that, in the absence of silicon-containing grain mantles, $\mathrm{SiO}$ line emission in steady-state planar C-type shock waves arises predominantly from cool postshock gas, close to maximum compression, with negligible emission from the precursor. Except at the lowest shock speeds, the $\mathrm{SiO}$ emission is optically thick and close to LTE for $4 \leq j_{\text {up }} \leq 7$. The relative line intensities, as functions of $j_{\text {up }}$, together with the absolute 5-4 line intensity, provide good diagnostics of the shock parameters, $n_{\mathrm{H}}$ and $v_{\mathrm{s}}$. The influence of the viewing angle and transverse magnetic field strength is found to be relatively minor over the typical ranges of their values.

Our shock models provide good fits to both the relative and absolute $\mathrm{SiO}$ intensities in the molecular outflows L1157 and L1448; the $\mathrm{SiO}$ fractional abundance is deduced to be in the range $4 \times 10^{-8} \lesssim n(\mathrm{SiO}) / n_{\mathrm{H}} \lesssim 3 \times 10^{-7}$. The emission regions of the shock wave are much colder, more optically thick, and have 10 to 100 times greater $\mathrm{SiO}$ column density than estimated previously from optically thin LVG slab models (Nisini et al. 2007). Our results are in line with a recent analysis of interferometric maps of the HH212 jet (Cabrit et al. 2007), demonstrating that the $\mathrm{SiO}$ emission is optically thick and close to LTE, with an intrinsic peak brightness temperature of approximately $50 \mathrm{~K}$. On the other hand, the line profiles predicted by our planar C-type shocks are typically 10 times narrower than observed in L1157 and L1448, suggesting that the single-dish beam includes shocks with various inclinations and speeds, and/or mixing layers. Detailed modelling of the line ratios as functions of velocity, with a careful correction for differing beam widths, would be needed to clarify this issue.

Whilst the grid of models presented here is intended to provide a guide to interpreting observations of outflow sources, it should be recalled that the dynamical timescales which characterize these regions are often too short to enable C-type shock waves to attain their steady-state structure (Chièze et al. 1998; Lesaffre et al. 2004). The presence of an embedded J-type discontinuity has then to be considered when modelling specific sources. Studies of the outflow source in Orion (Le Bourlot et al. 2002), of jets associated with low-mass star formation (Giannini et al. 2004, 2006; McCoey et al. 2004), and of the supernova remnant IC 443 (Cesarsky et al. 1999) have shown that the rovibrational line spectrum of $\mathrm{H}_{2}$ can be reproduced successfully by hybrid shock waves, but not by pure C- or J-type shocks. The influence of the discontinuity on the C-component (magnetic precursor), and hence on the formation of $\mathrm{SiO}$ and its emission line spectrum, becomes important for ages smaller than the flow time to maximum compression.

The existence of Si-containing grain mantles is another circumstance that would significantly modify the intensities of $\mathrm{SiO}$ lines and their profiles. The fractional abundance of $\mathrm{SiO}$ in the warm shock precursor would be enhanced, compared with the models considered here, in which $\mathrm{Si}$ is sputtered exclusively from olivine grain cores. The effects of non-steady C-type 
shocks and Si-containing mantles will be considered in a forthcoming paper.

Acknowledgements. Antoine Gusdorf and the University of Durham acknowledge the support of the European Commission under the Marie Curie Research Training Network "The Molecular Universe" MRTN-CT-2004-512302. We thank Brunella Nisini for helpful correspondence relating to the $\mathrm{SiO}$ observations of Nisini et al. (2007). We thank also Paul Goldsmith and Laurent Pagani for information regarding SWAS and Odin observations of $\mathrm{O}_{2}$.

\section{References}

Anders, E., \& Grevesse, N. 1989, Geochim. Cosmochim. Act., 53, 197

Bachiller, R., Martín-Pintado, J., \& Fuente, A. 1991, A\&A, 243, L21

Benedettini, M., Viti, S., Codella, C., et al. 2007, MNRAS, 381, 1127

Cabrit, S., Codella, C., Gueth, F., et al. 2007, A\&A, 468, L29

Caselli, P., Hartquist, T. W., \& Havnes, O. 1997, A\&A, 322, 296

Cesarsky, D., Cox, P., Pineau des Forêts, G., et al. 1999, A\&A, 348, 945

Chièze, J.-P., Pineau des Forêts, G., \& Flower, D. R. 1998, MNRAS, 295, 672

Ciolek, G. E., Roberge, W. G., \& Mouschovias, T. Ch. 2004, ApJ, 610, 781

Codella, C., Bachiller, R., \& Reipurth, B. 1999, A\&A, 343, 585

Crutcher, R. M. 1999, ApJ, 520, 706

Curiel, S., Torrelles, J. M., Rodríguez, L. F., Gómez, J. F., \& Anglaga, G. 1999, ApJ, 527, 310

Dayou, F., \& Balança, C. 2006, A\&A, 459, 297

Dickinson, D. F. 1972, ApJ, 175, L43

Draine, B. T., Roberge, W. G., \& Dalgarno, A. 1983, ApJ, 264, 485

Dutrey, A., Guilloteau, S., \& Bachiller, R. 1997, A\&A, 325, 758

Field, D., May, P. W., Pineau des Forêts, G., \& Flower, D. R. 1997, MNRAS, 285,839

Flower, D. R., \& Pineau des Forêts, G. 1994, MNRAS, 268, 724

Flower, D. R., \& Pineau des Forêts, G. 1995, MNRAS, 275, 1049

Flower, D. R., \& Pineau des Forêts, G. 2003, MNRAS, 343, 390

Flower, D. R., Pineau des Forêts, G., Field, D., \& May, P. W. 1996, MNRAS, 280,447

Flower, D. R., Le Bourlot, J., Pineau des Forêts, G., \& Cabrit, S. 2003, Ap\&SS, 287,183

Giannini, T., McCoey, C., Caratti o Garatti, A., et al. 2004, A\&A, 419, 999

Giannini, T., McCoey, C., Nisini, B., et al. 2006, A\&A, 459, 821

Goldreich, P., \& Kwan, J. 1974, ApJ, 189, 441

Graff, M. M. 1989, ApJ, 339, 239

Gueth, F., Guilloteau, S., \& Bachiller, R. 1998, A\&A, 333, 287

Guillet, V., Pineau des Forêts, G., \& Jones, A. 2007, A\&A, 476, 263

Guilloteau, S., Bachiller, R., Fuente, A., \& Lucas, R. 1992, A\&A, 265, L49

Hummer, D. G., \& Rybicki, G. B. 1982, ApJ, 254, 767

Jiménez-Serra, I., Martín-Pintado, J., Rodríguez-Franco, A., \& Marcelino, N. 2004, ApJ, 603, L49

Jiménez-Serra, I., Martín-Pintado, J., Rodríguez-Franco, A., \& Martín, S. 2005, ApJ, 627, L121

Langer, W. D., \& Glassgold, A. E. 1990, ApJ, 352, 123

Larsson, B., Liseau, R., Pagani, L., et al. 2007, A\&A, 466, 999

Le Bourlot, J., Pineau des Forêts, G., Flower, D. R., \& Cabrit, S. 2002, MNRAS, 332,985

Lefloch, B., Castets, A., Cernicharo, J., \& Loinard, L. 1998, ApJ, 504, L109

Le Picard, S. D., Canosa, A., Pineau des Forêts, G., Rebrion-Rowe, C., \& Rowe, B. R. 2001, A\&A, 372, 1064

Lesaffre, P., Chièze, J.-P., Cabrit, S., \& Pineau des Forêts, G. 2004, A\&A, 427, 157

Le Teuff, Y. H., Millar, T. J., \& Markwick, A. J. 2000, A\&AS, 146, 157

Li, A., \& Draine, B. T. 2001, ApJ, 554, 778

Martín-Pintado, J., Bachiller, R., \& Fuente, A. 1992, A\&A, 254, 315

Mathis, J. S., Rumpl, W., \& Nordsieck, K. H. 1977, ApJ, 217, 425

May, P. W., Pineau des Forêts, G., Flower, D. R., et al. 2000, MNRAS, 318, 809

McCoey, C., Giannini, T., Flower, D. R., \& Caratti o Garatti, A. 2004, MNRAS, 353,813

Neufeld, D. A., \& Kaufman, M. J. 1993, ApJ, 418, 263

Nisini, B., Codella, C., Giannini, T., et al. 2007, A\&A, 462, 163

Pagani, L., Olofsson, A. O. H., Bergman, P., et al. 2003, A\&A, 402, L77

Schilke, P., Walmsley, C. M., Pineau des Forêts, G., \& Flower, D. R. 1997, A\&A, 321, 293 (Sch97)

Schoier, F. L., Van der Tak, F. F. S., Van Dishoeck, E. F., \& Black, J. H. 2005, A\&A, 432, 369

Surdej, J. 1977, A\&A, 60, 303

Turner, B. E., Chan, K., Green, S., \& Lubowich, D. A. 1992, ApJ, 321, 293

Van der Tak, F. F. S., Black, J. H., Schöier, F. L., Jansen, D. J., \& van Dishoeck, E. F. 2007, A\&A, 468, 627
Weingartner, J. C., \& Draine, B. T. 2001, ApJS, 134, 263

Wilson, R. W., Penzias, A. A., Jefferts, K. B., Kutner, M., \& Thaddeus, P. 1971, ApJ, 167, L97

Wrathmall, S. A., Gusdorf, A., \& Flower, D. R. 2007, MNRAS, 382, 133

Zachariah, M. R., \& Tsang, W. 1995, J. Phys. Chem., 99, 5308

Zhang, Q., Ho, P. T. P., Wright, M. C. H., \& Wilner, D. J. 1995, ApJ, 451, L71

\section{Appendix A: SiO radiative transfer}

\section{A.1. Photon escape probabilities}

The $\mathrm{SiO}$ rotational level populations and excitation temperatures in our planar shock models are calculated by means of a large velocity gradient (LVG) method. This approximate treatment assumes that, owing to the macroscopic velocity field and resulting Doppler shifts, emitted line photons are either re-absorbed locally or escape to infinity. The escape probability of a line photon in a given direction $\hat{s}$ is then (see Surdej 1977, for a pedagogical derivation):

$\beta_{s}=\frac{1-\mathrm{e}^{-\tau_{s}}}{\tau_{s}}$

where the "LVG optical depth" $\tau_{s}$ is defined as

$\tau_{s}=\frac{h c}{4 \pi} \frac{n_{\mathrm{l}}}{\partial(\boldsymbol{v} \cdot \hat{\boldsymbol{s}}) / \partial s} B_{\mathrm{lu}}\left(1-\frac{g_{\mathrm{l}} n_{\mathrm{u}}}{g_{\mathrm{u}} n_{\mathrm{l}}}\right)$,

with $\partial(\boldsymbol{v} \cdot \hat{\boldsymbol{s}}) / \partial s$ the radial velocity gradient along direction $\hat{\boldsymbol{s}}, n_{l}$ and $n_{u}$ the number density of molecules in the lower and upper levels of the transition, respectively, $g_{1}$ and $g_{\mathrm{u}}$ the corresponding statistical weights, and $B_{\mathrm{lu}}$ the Einstein coefficient for stimulated absorption. The mean intensity of the radiation field at the local frequency $v$ of the transition, averaged over all angles, may then be expressed in terms of local quantities only as

$\bar{J}_{v}=S_{v}(1-\bar{\beta})+I_{\mathrm{c}} \bar{\beta}$,

where $\bar{\beta}=\int \beta_{s} \mathrm{~d} \Omega / 4 \pi$ is the escape probability averaged over all solid angles; $I_{\mathrm{c}}$ is the mean intensity of the continuum radiation field, taken to be a blackbody (Planck) function $B_{v}(T)$ at the temperature of the cosmic background, $T_{\mathrm{bg}}=2.73 \mathrm{~K}$; and $S_{v}=B_{v}\left(T_{\mathrm{ex}}\right)$ is the local source function, where the excitation temperature $T_{\mathrm{ex}}$ of the transition is defined through $n_{\mathrm{u}} / n_{1} \equiv g_{\mathrm{u}} / g_{1} \exp \left(-h v / k_{\mathrm{B}} T_{\text {ex }}\right)$. Two expressions have been used for the average escape probability $\bar{\beta}$ :

- that of Neufeld \& Kaufman (1993):

$$
\bar{\beta}_{\text {plane }}=\frac{1}{1+3 \tau_{\perp}}
$$

where $\tau_{\perp}$ is the LVG opacity in the $z$-direction, normal to the shock front. This approximation to $\bar{\beta}$ is accurate for a planeparallel flow, where $\partial(\boldsymbol{v} \cdot \hat{\boldsymbol{s}}) / \partial s=\mu^{2}\left(\mathrm{~d} v_{z} / \mathrm{d} z\right)$ and $\tau_{s}=\tau_{\perp} / \mu^{2}$ (with the usual notation $\mu=\cos \theta=\hat{\boldsymbol{s}} \cdot \hat{z}$ ).

- an isotropic approximation:

$$
\bar{\beta}_{\text {isotropic }}=\beta_{\perp}=\frac{1-\mathrm{e}^{-\tau_{\perp}}}{\tau_{\perp}} .
$$

The first expression was ultimately adopted in the present study for consistency with our one-dimensional shock geometry, whereas the second was used by $\operatorname{Sch} 97$. Since $\bar{\beta}$ is always smaller in the plane-parallel case (owing to the reduced velocity gradients at small $\mu$ ), photon trapping is more efficient and the excitation temperatures are increased compared to the isotropic approximation. Thus, adopting the expression of 


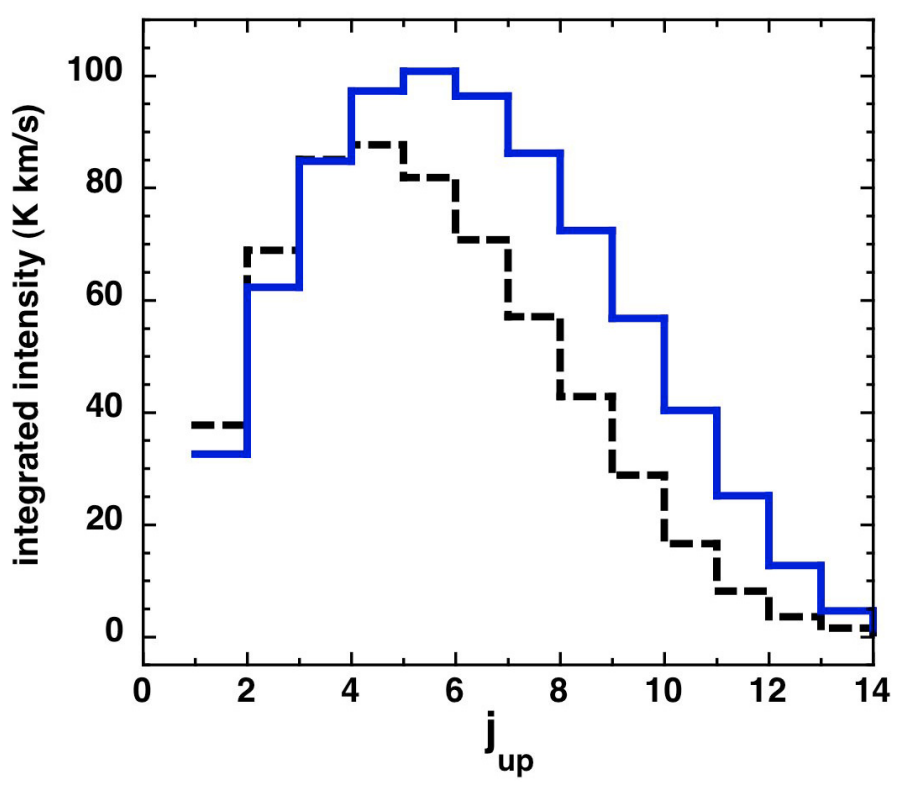

Fig. A.1. The integrated intensities of the rotational transitions of $\mathrm{SiO}$. Full curves correspond to the Neufeld \& Kaufman (1993) expression (A.4) for the escape probability in a plane-parallel flow, dashed curves to the isotropic case, Eq. (A.5). The model parameters are $n_{\mathrm{H}}=10^{5} \mathrm{~cm}^{-3}, B=200 \mu \mathrm{G}$, and $v_{\mathrm{s}}=30 \mathrm{~km} \mathrm{~s}^{-1}$.

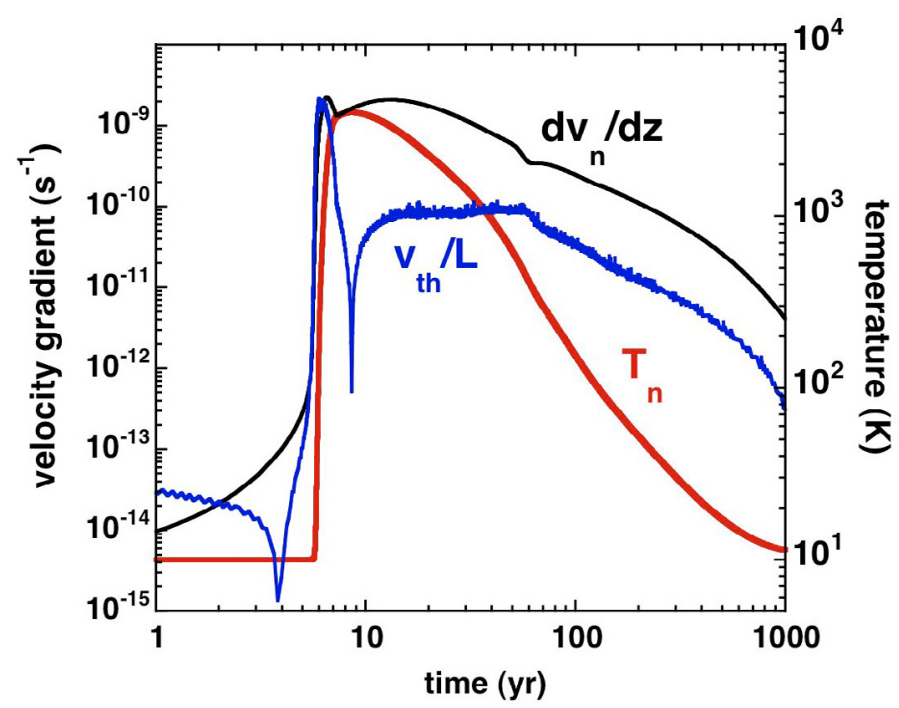

Fig. A.2. Consistency of the LVG method: velocity gradient criterion (A.6). The model parameters are $n_{\mathrm{H}}=10^{5} \mathrm{~cm}^{-3}, B=200 \mu \mathrm{G}$, and $v_{\mathrm{s}}=30 \mathrm{~km} \mathrm{~s}^{-1}$.

Neufeld \& Kaufman (1993) leads to larger integrated SiO line intensities; this effect is illustrated in Fig. A.1 for our reference shock model. It is marginally significant for small and large values of the rotational quantum number but reaches a factor 3 for $j_{\text {up }} \approx 12$.

A necessary condition for the local LVG approximation to be valid is that, over the characteristic distance $L$ where physical conditions vary, the velocity shift $\Delta v$ arising from the velocity gradient should be larger than the local line thermal width $v_{\text {th }}$. Then, line photons are re-absorbed only within a region of size $<L$, where physical conditions and $\mathrm{SiO}$ excitation are uniform. This criterion can be rewritten as

$\left|\mathrm{d} v_{z} / \mathrm{d} z\right|>v_{\mathrm{th}} / L$ where

$v_{\mathrm{th}}=\sqrt{\frac{8 k_{\mathrm{B}} T_{\mathrm{n}}}{\pi m}}$,

$T_{\mathrm{n}}$ is the temperature of the neutral fluid, and $m$ is the mass of the molecule.

In Fig. A. 2 we compare the left and right hand sides of (A.6) for our reference shock model, using $L \approx T_{\mathrm{n}} /\left|\mathrm{d} T_{\mathrm{n}} / \mathrm{d} z\right|$ as a characteristic distance. The LVG criterion may be seen to be verified throughout the cooling flow of the shock wave, where the bulk of $\mathrm{SiO}$ emission arises. It is not verified in the far postshock region, where the computed velocity gradient tends to zero; but this region makes a negligible contribution to the line flux, owing to the low escape probabilities.

\section{A.2. Numerical implementation}

In the diatomic $\mathrm{SiO}$ molecule, electric dipole transitions take place only between adjacent rotational levels of the ground vibrational state $(\Delta j= \pm 1$ with $j$ the rotational quantum number), while collisionally induced transitions can, in principle, connect any pair of levels (in practice they become less probable with increasing level separation). The evolution of the population density $n_{i}$ of level $i$ may thus be written in the following matrix form:

$$
\begin{aligned}
\frac{\mathrm{d} n_{i}}{\mathrm{~d} t}= & n_{i+1}\left(A_{i+1, i}+B_{i+1, i} \bar{J}_{i, i+1}\right) \\
& -n_{i}\left(A_{i, i-1}+B_{i, i+1} \bar{J}_{i, i+1}+B_{i, i-1} \bar{J}_{i-1, i}\right) \\
& +n_{i-1} B_{i-1, i} \bar{J}_{i-1, i} \\
& +\sum_{\text {coll }} \sum_{j \neq i} n_{\mathrm{coll}}\left(n_{j} C_{j i}-n_{i} C_{i j}\right),
\end{aligned}
$$

where $n_{\text {coll }}$ is the number density of each collisional partner (here $\mathrm{H}_{2}$ and $\left.\mathrm{He}\right), C_{i j}$ is the collisional rate coefficient from level $i$ to level $j$, and $A$ and $B$ denote the Einstein coefficients for spontaneous and induced radiative transitions. Two approaches to solving for the level populations may be taken:

- Integrate the set of Eqs. (A.7) in parallel with the dynamical, thermal, and chemical rate equations of the shock wave, as is done currently for the $\mathrm{H}_{2}$ molecule (Le Bourlot et al. 2002).

- Assume local statistical equilibrium, i.e. set the lhs of Eqs. (A.7) to zero, and solve a posteriori the resulting algebraic equations by matrix inversion, using the physical structure provided by the MHD shock code. Since the radiative terms $\bar{J}$ depend indirectly on the level populations through the escape probabilities and excitation temperatures (Eq. (A.3)), the procedure must be iterated, updating $\bar{J}$ with values of $\bar{\beta}$ and $T_{\text {ex }}$ obtained from the previous iteration.

The former approach is preferable, particularly when the molecule under consideration is an important shock coolant (e.g. $\mathrm{H}_{2}$ ) - which is not the case of $\mathrm{SiO}$. Furthermore, the flow time in the $\mathrm{SiO}$ emission zone is sufficiently long that the assumption of local statistical equilibrium is justified. Accordingly, we adopted the latter approach in the present work. It is common to most applications of the LVG method to astrophysical problems (e.g. Sch97, Neufeld \& Kaufman 1993) and has the advantage of being less demanding in CPU time. For more significant coolants, such as $\mathrm{CO},{ }^{13} \mathrm{CO}$, and $\mathrm{H}_{2} \mathrm{O}$, the rate of cooling was computed in parallel with the shock dynamics, using the cooling 
functions calculated by Neufeld \& Kaufman (1993), by means of the LVG method.

Note that Eqs. (A.7) may be put in a different (matrix) form by expressing explicitly $\bar{J}$ as a function of $B_{v}\left(T_{\mathrm{ex}}\right)$ and $I_{\mathrm{c}}$. Using the standard relationships between Einstein coefficients, and the definition of $T_{\text {ex }}$ in terms of $n_{j}$ and $n_{j+1}$, all terms involving $T_{\text {ex }}$ cancel from the equations, leaving radiative terms which are proportional to the $\bar{\beta}$ 's (see Goldreich \& Kwan 1974):

$$
\begin{aligned}
\frac{\mathrm{d} n_{i}}{\mathrm{~d} t}= & +n_{i+1} \bar{\beta}_{i, i+1}\left(A_{i+1, i}+B_{i+1, i} I_{\mathrm{c}}\right) \\
& -n_{i}\left(\bar{\beta}_{i-1, i} A_{i, i-1}+\bar{\beta}_{i, i+1} B_{i, i+1} I_{\mathrm{c}}+\bar{\beta}_{i-1, i} B_{i, i-1} I_{\mathrm{c}}\right) \\
& +n_{i-1} \bar{\beta}_{i-1, i} B_{i-1, i} I_{\mathrm{c}} \\
& +\sum_{\text {coll }} \sum_{j \neq i} n_{\mathrm{coll}}\left(n_{j} C_{j i}-n_{i} C_{i j}\right) .
\end{aligned}
$$

Although the two formulations are equivalent and converge to the same solution, we have found that inversion of the latter matrix encounters numerical instabilities and convergence problems at high optical depths, possibly due to round-off errors in the (vanishingly small) $\bar{\beta}$ terms. In the first formulation, the radiative elements of the matrix are never zero, even at high opacity, and we obtain much better convergence and accuracy. Hence we have adopted (A.7) in the present calculations. The "lambdaiteration" is terminated when we reach convergence of the level populations to 1 part in $10^{4}$.

Our code was tested thoroughly against the routine used by Sch97, in the case $\bar{\beta}=\beta_{\perp}$, and against the web-based online version of RADEX (www.sron.rug.nl/ vdtak/radex/radex.php), which uses yet another expression for the escape probability, valid for a turbulent homogeneous sphere (Van der Tak et al. 2007). Discrepancies never exceeded a few percent.

The MHD shock code provides the physical and chemical profiles (of the temperatures, densities, velocities, and abundances) which are required in order to apply the LVG technique. For $\mathrm{SiO}$, we used the rate coefficients for collisional de-excitation published by Turner et al. (1992), for which the collision partner is ground state para- $\mathrm{H}_{2}$. These data are available for rotational quantum numbers $0 \leq J \leq 20$ and for kinetic temperatures $T=20,40,70,100,150,200,250,300 \mathrm{~K}$ and are interpolated to intermediate values of $T$. We made use also of the extrapolated data of Schöier et al. (2005), which extend to $J=40$ and $T=2000 \mathrm{~K}$. Subsequent calculations, using the rate coefficients of Dayou \& Balança (2006) for collisions of $\mathrm{SiO}$ with para- $\mathrm{H}_{2}$, have shown that the rotational line intensities are insensitive to these collision rates because the lines are formed under conditions which approach LTE. For collisions of $\mathrm{SiO}$ with $\mathrm{He}$, we used the rate coefficients of Dayou $\&$ Balança (2006), for $J \leq 26$ and kinetic temperatures in the range $10 \leq T \leq 300 \mathrm{~K}$. At temperatures higher than the maximum for which the rate coefficients were calculated, we assumed that they remain constant. Upwards (excitation) rate coefficients were obtained from detailed balance.

The Einstein A-values, and the rotational constant $B_{0}=$ $21711.967 \mathrm{MHz}$ (corresponding to $h B_{0} / k_{\mathrm{B}}=1.042 \mathrm{~K}$ ) for the ground vibrational state of ${ }^{28} \mathrm{Si}^{16} \mathrm{O}$ were taken from the NIST database (wWw . nist.gov/data/).

\section{A.3. Emergent line intensities and radiation temperatures}

Each layer of the shock wave, of thickness $|\Delta z|$, elementary surface $\Delta S$, and velocity $v_{z}$, emits the following luminosity (in erg $\mathrm{s}^{-1}$ ) over all directions in a given transition $j+1 \rightarrow j$ of frequency $v=2 B_{0}(j+1)$ :

$F_{v}\left(v_{z}\right)=\bar{\beta} h v n_{j+1} A_{j+1, j}|\Delta z| \Delta S$.

In order to compute line profiles, however, we need to evaluate the specific intensity per unit solid angle, frequency interval, and projected emitting area (in erg $\mathrm{cm}^{-2} \mathrm{~s}^{-1} \mathrm{~Hz}^{-1} \mathrm{sr}^{-1}$ ). Following Sch97, we assume that the shock is viewed face-on, i.e. in the $z$-direction, normal to the shock front. The intensity is

$I_{\perp}\left(v_{z}\right)=\frac{\beta_{\perp}}{4 \pi} h v n_{j+1} A_{j+1, j} \frac{|\Delta z|}{\left|\Delta v_{z}\right|}$,

where $\left|\Delta v_{z}\right|$ is the Doppler width of the layer, viewed along the $z$-axis:

$\left|\Delta v_{z}\right|=\frac{v}{c}\left|\Delta v_{z}\right|=\frac{v}{c}|\Delta z| \times\left|\mathrm{d} v_{z} / \mathrm{d} z\right|$.

Consequently the intensity becomes

$I_{\perp}\left(v_{z}\right)=\frac{h c}{4 \pi} \frac{\beta_{\perp}}{\left|\mathrm{d} v_{z} / \mathrm{d} z\right|} n_{j+1} A_{j+1, j}$.

Noting that $\beta_{\perp}=\left(1-\mathrm{e}^{-\tau_{\perp}}\right) / \tau_{\perp}$ and using the definition of $\tau$ in Eq. (A.2) as well as the relationships between $A$ and $B$-Einstein coefficients, one obtains the alternative, simpler expression

$I_{\perp}\left(v_{z}\right)=B_{v}\left(T_{\mathrm{ex}}\right)\left(1-\mathrm{e}^{-\tau_{\perp}}\right)$

which corresponds to the well-known radiative transfer result for a uniform slab of excitation temperature $T_{\mathrm{ex}}$ and opacity $\tau_{\perp}$.

In order to compare with actual observed $\mathrm{SiO}$ spectra, one needs to add the cosmic background, and take into account the ON-OFF subtraction applied to radio spectra (to remove atmospheric noise). The cosmic background at the ON position and velocity $v_{z}$ is $B_{v}\left(T_{\mathrm{bg}}\right) \exp \left(-\tau_{\perp}\right)$, due to attenuation by the layer, while at the OFF position it is simply $B_{v}\left(T_{\mathrm{bg}}\right)$. The observed intensity is thus

$$
\begin{aligned}
I_{\perp}^{\mathrm{obs}}\left(v_{z}\right)=\mathrm{ON}-\mathrm{OFF} & =\left[B_{v}\left(T_{\mathrm{ex}}\right)-B_{v}\left(T_{\mathrm{bg}}\right)\right]\left(1-\mathrm{e}^{-\tau_{\perp}}\right) \\
& =I_{\perp}\left(v_{z}\right) \times\left[1-B_{v}\left(T_{\mathrm{bg}}\right) / B_{v}\left(T_{\mathrm{ex}}\right)\right] .
\end{aligned}
$$

Finally, we convert the observed specific intensity of the layer to a line radiation temperature $T_{\mathrm{R}}$ (in kelvin) using the definition

$T_{\mathrm{R}} \equiv \frac{I^{\mathrm{obs}} c^{2}}{2 k_{B} v^{2}}$

which is standard in radioastronomy. Once radiation temperatures are calculated for each shock layer, and thus each $v_{z}$, the line profile is integrated over $v_{z}$ to yield integrated line intensities, $T \mathrm{~d} V$, in $\mathrm{K} \mathrm{km} \mathrm{s}^{-1}$. Note that the specific intensities, radiation temperatures and $T \mathrm{~d} V$ are valid for a shock that entirely fills the observing beam. Otherwise, the observed brightness temperature has to be reduced by an appropriate "surface filling factor", $f \approx \Delta S /$ (beam area).

\section{A.4. Line profile at arbitrary inclinations}

In the general case of an arbitrary viewing angle, $\mu=\cos \theta$, the term $\tau_{\perp}$ in Eq. (A.13) is replaced by the LVG opacity in the chosen direction, $\tau(\mu)=\tau_{\perp} / \mu^{2}$. Therefore

$T_{\mathrm{R}}(\mu)=T_{\mathrm{R} \perp} \frac{\left(1-\mathrm{e}^{-\tau_{\perp} / \mu^{2}}\right)}{\left(1-\mathrm{e}^{-\tau_{\perp}}\right)}$ 
Table B.1. The numerical values of the coefficients, $a_{i}(X)$, of the polynomial fits to the fractions of $X \equiv \mathrm{Fe}$, Si and Mg sputtered from olivine grains. Numbers in parentheses are powers of 10.

\begin{tabular}{lcccccc}
\hline \hline$X$ & $a_{0}$ & $a_{1}$ & $a_{2}$ & $a_{3}$ & $a_{4}$ & $a_{5}$ \\
\hline \hline $\mathrm{Mg}$ & -0.5322 & 0.09172 & -0.006035 & 0.0001879 & $-2.746(-06)$ & $1.522(-08)$ \\
$\mathrm{Si}$ & -0.4147 & 0.07364 & -0.004953 & 0.0001560 & $-2.279(-06)$ & $1.250(-08)$ \\
$\mathrm{Fe}$ & -0.8579 & 0.14681 & -0.009607 & 0.0002979 & $-4.349(-06)$ & $2.413(-08)$ \\
\hline
\end{tabular}

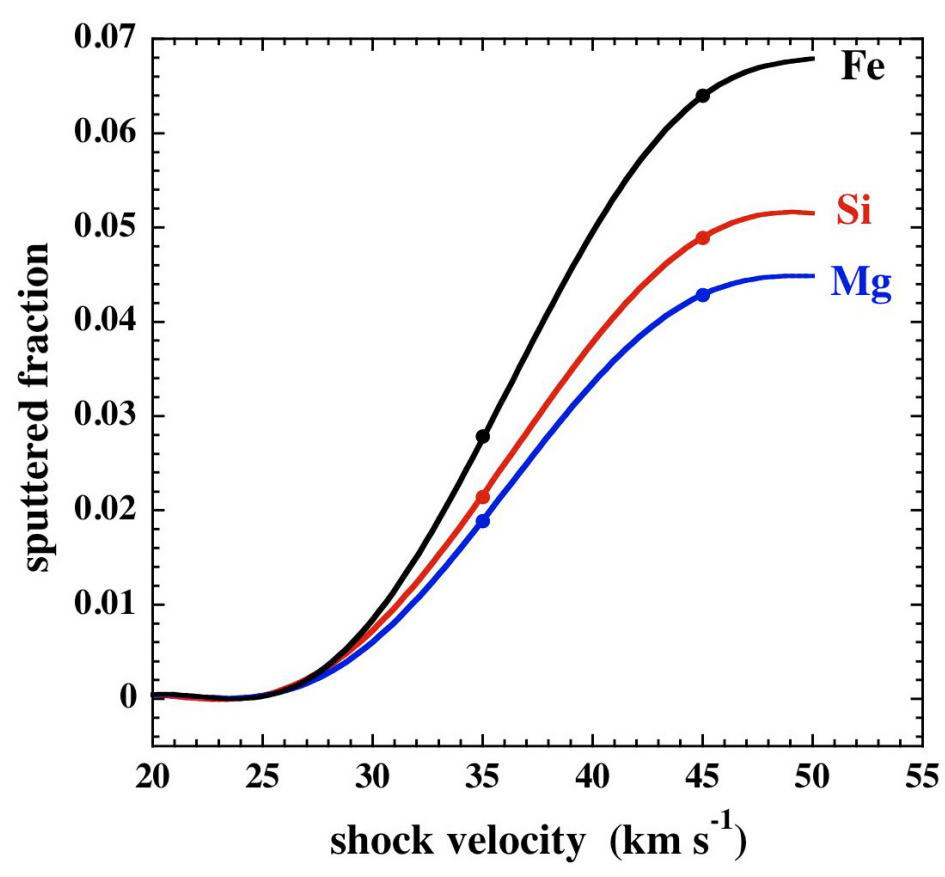

Fig. B.1. Fits of the fractions of $\mathrm{Fe}, \mathrm{Si}$ and $\mathrm{Mg}$ sputtered from olivine grains, as functions of the shock speed. The points on the curves indicate the limiting speeds of steady-state C-type shock waves, for preshock gas densities $n_{\mathrm{H}}=10^{5}$ and $10^{6} \mathrm{~cm}^{-3}$, with the lower speed corresponding to the higher density. The parameters of the fits to the curves are given in Table B.1.
Thus, $T_{\mathrm{R}}$ will be multiplied by $1 / \mu^{2}$ in the optically thin regime and will be unchanged in the optically thick regime. At the same time, the velocity of the layer, $v_{z}$, is replaced by its projection along the photon path, $\mu v_{z}$, so the line profile becomes narrower. A view which is not face-on will leads to a larger $T \mathrm{~d} V$ for optically thin lines, and to a smaller $T \mathrm{~d} V$ for optically thick lines (assuming that the source still fills the beam entirely). A quantitative evaluation of this effect, for our reference shock model, is presented in Sect. 4.4.

\section{Appendix B: Fractions of Fe, Si and Mg sputtered from olivine}

In Sect. 3.2, we presented and discussed the sputtering of Fe, $\mathrm{Si}$ and $\mathrm{Mg}$ from grains composed of olivine $\left(\mathrm{MgFeSiO}_{4}\right)$. In Fig. B.1, we show the numerical fits to these results, as functions of the shock speed; they are practically independent of the preshock gas density. Also shown in this figure are the limiting speeds of steady-state C-type shock waves for preshock densities $n_{\mathrm{H}}=10^{5}$ and $10^{6} \mathrm{~cm}^{-3}$; the limiting speed is lower for the higher density. The limit is associated with the thermal runaway which occurs, owing to the collisional dissociation of $\mathrm{H}_{2}$ within the shock wave. The numerical values of the coefficients of the polynomial fits, of the form

$y(\mathrm{X})=\sum_{i=0}^{5} a_{i}(\mathrm{X}) v_{\mathrm{s}}^{i}$

are given in Table B.1; the shock speed, $v_{\mathrm{s}}$, is in $\mathrm{km} \mathrm{s}^{-1}$. We have assumed that the magnetic field parameter $b=1$.

\section{Appendix C: Initial gas-phase abundance of $\mathrm{O}_{2}$}

We present, in Figs. C.1-C.4, the results corresponding to those in Figs. 4, 7, 8 and 12, respectively, of the main text, but derived from our secondary grid of models, in which $n\left(\mathrm{O}_{2}\right) / n_{\mathrm{H}}=1.0 \times$ $10^{-7}$ initially in the gas phase but the excess oxygen is in form of $\mathrm{H}_{2} \mathrm{O}$ ice, rather than $\mathrm{O}_{2}$ ice as in our primary grid. We recall that the initial fractional abundance of $\mathrm{O}_{2}$ in chemical equilibrium is $n\left(\mathrm{O}_{2}\right) / n_{\mathrm{H}} \approx 10^{-5}$.

In these models, $\mathrm{O}_{2}$ is never abundant in the gas phase, and $\mathrm{Si}$ oxidation occurs only in reaction 2 , with $\mathrm{OH}$. Thus, $\mathrm{SiO}$ formation is less efficient at intermediate shock speeds and not significant at $v_{\mathrm{s}}=25 \mathrm{~km} \mathrm{~s}^{-1}$. 

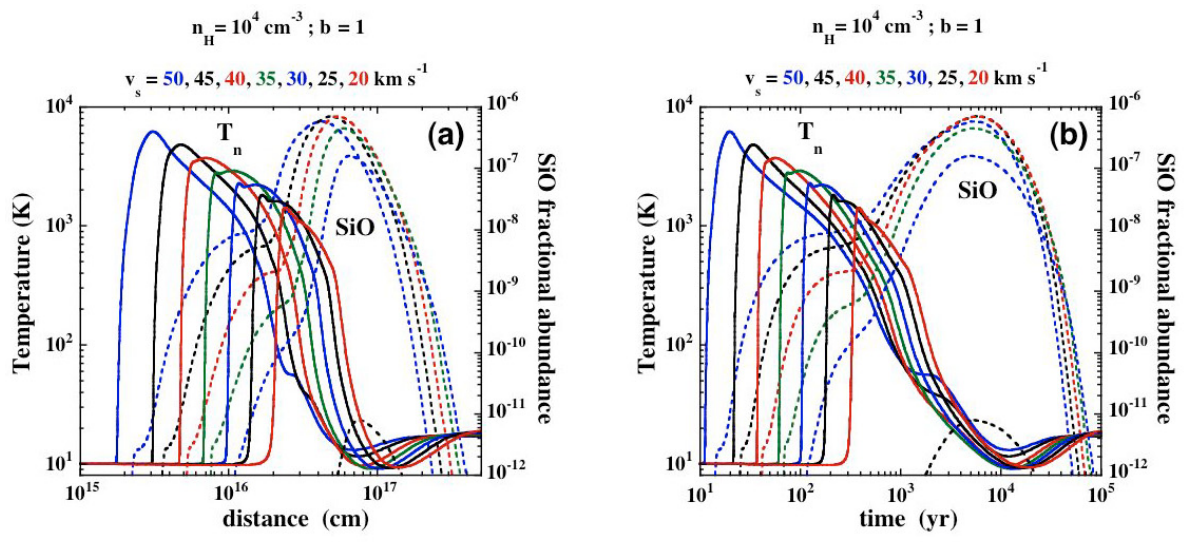

$\mathrm{n}_{\mathrm{H}}=10^{5} \mathrm{~cm}^{-3} ; \mathrm{b}=1$

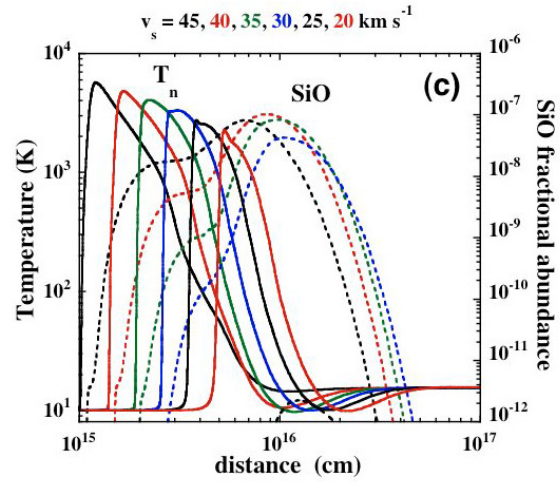

$$
\mathrm{n}_{\mathrm{H}}=10^{5} \mathrm{~cm}^{-3} ; \mathrm{b}=1
$$

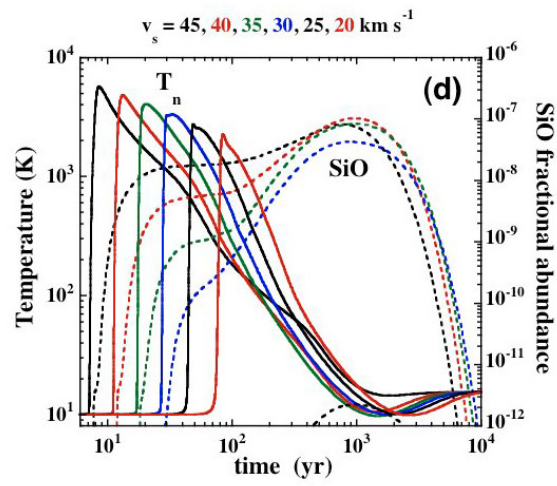

$\mathrm{n}_{\mathrm{H}}=10^{6} \mathrm{~cm}^{-3} ; \mathrm{b}=1$

$$
\mathrm{n}_{\mathrm{H}}=10^{6} \mathrm{~cm}^{-3} ; \mathrm{b}=1
$$
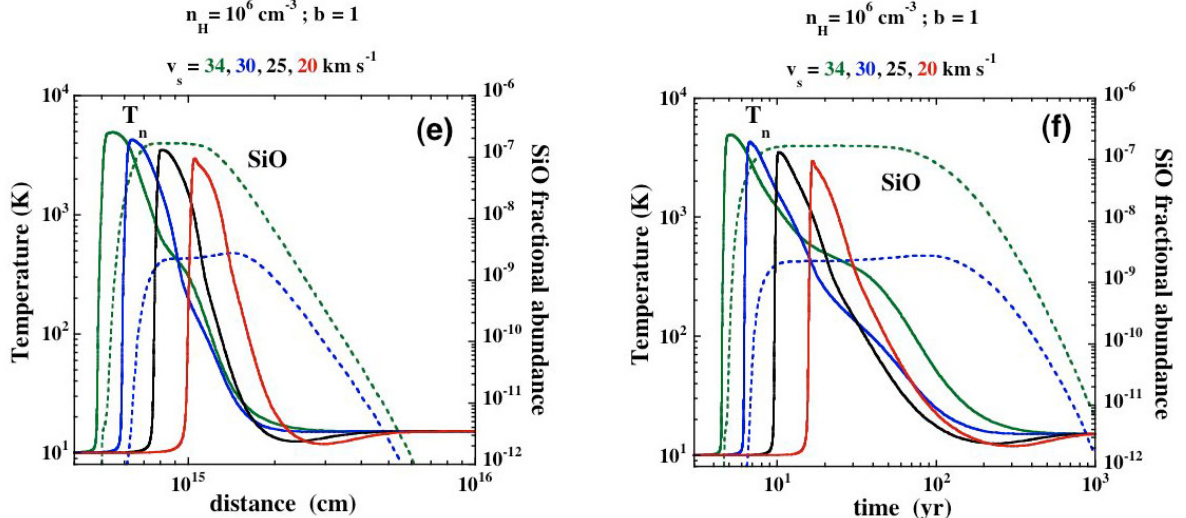

Fig. C.1. As Fig. 4, but assuming that the initial abundance of $\mathrm{O}_{2}$ ice is negligible (the second of the two scenarios described in Sect. 3.1). 

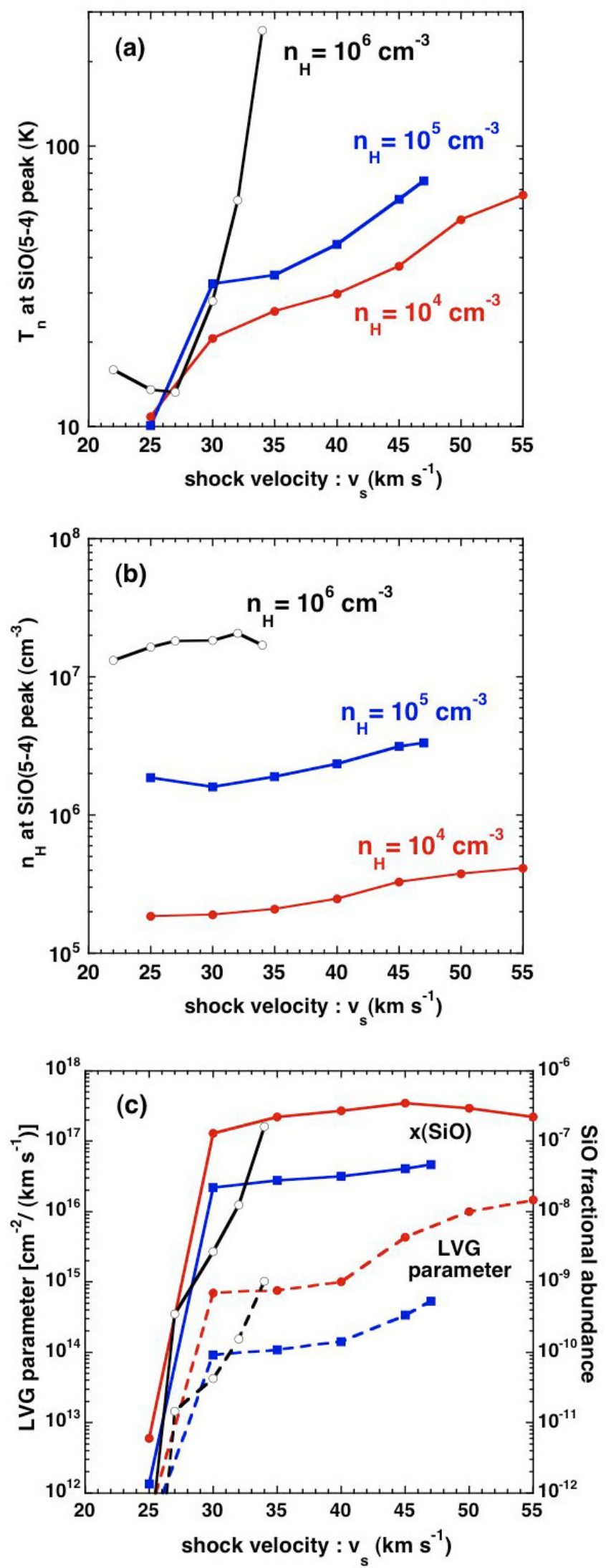

Fig. C.2. As Fig. 7, but assuming that the initial abundance of $\mathrm{O}_{2}$ ice is negligible (the second of the two scenarios described in Sect. 3.1).

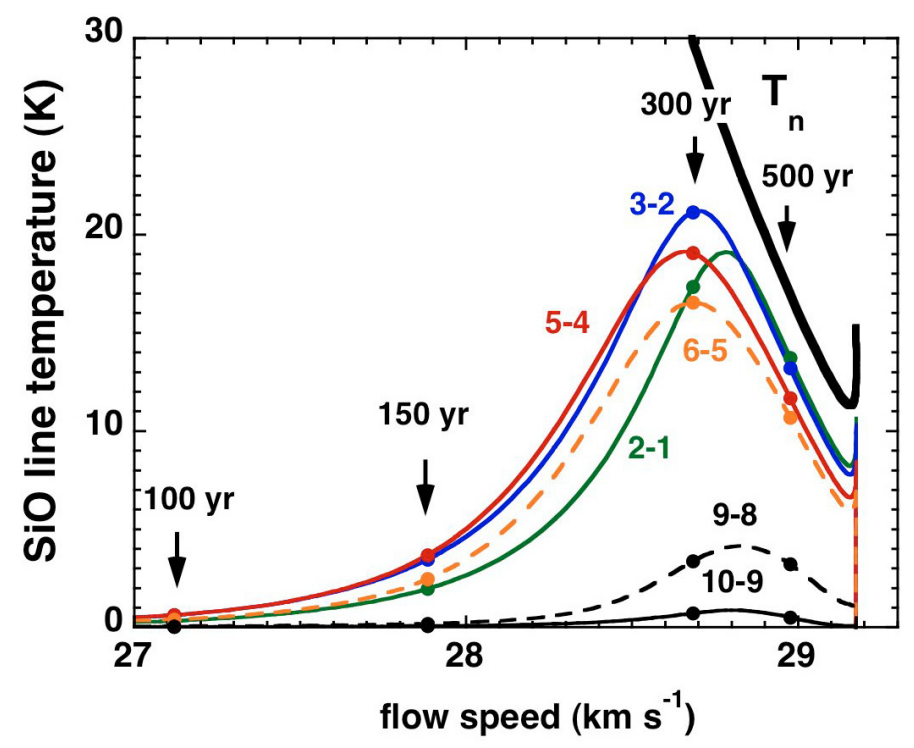

Fig. C.3. As Fig. 8, but assuming that the initial abundance of $\mathrm{O}_{2}$ ice is negligible (the second of the two scenarios described in Sect. 3.1). 
A. Gusdorf et al.: SiO line emission from C-type shock waves: interstellar jets and outflows
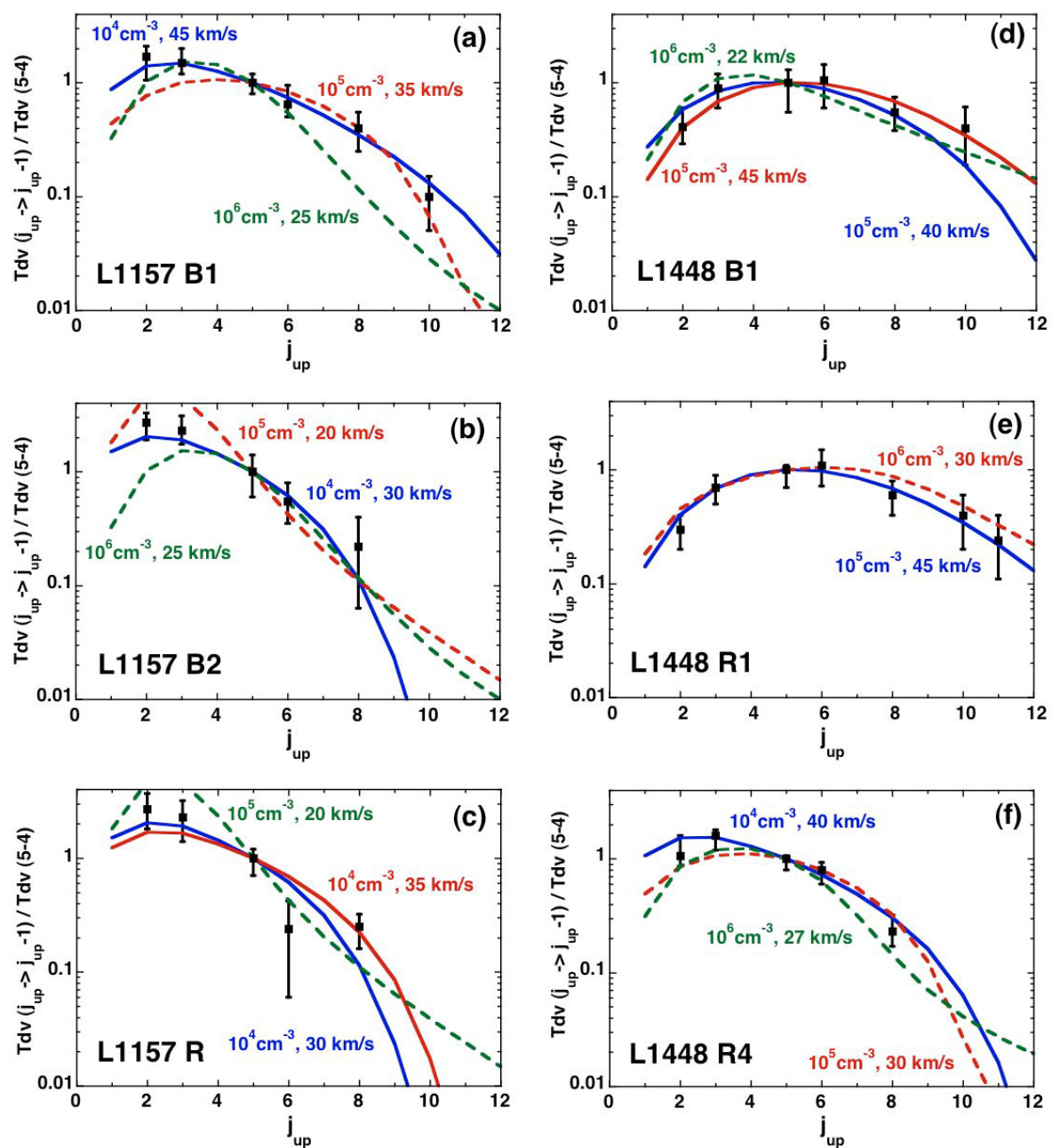

Fig. C.4. As Fig. 12, but assuming that the initial abundance of $\mathrm{O}_{2}$ ice is negligible (the second of the two scenarios described in Sect. 3.1). 